\title{
Turbo-Slice-and-Patch: An Algorithm for Metropolitan Scale VBR Video Streaming
}

\author{
Chun-Wai Kong, Jack Y. B. Lee, Senior Member, IEEE, Mounir Hamdi, Senior Member, IEEE, and \\ Victor O. K. Li, Fellow, IEEE
}

\begin{abstract}
In recent years, a number of sophisticated architectures have been proposed to provide video-on-demand (VoD) service using multicast transmissions. Compared to their unicast counterparts, these multicast VoD systems are highly scalable and can potentially serve millions of concurrent users. Nevertheless, these systems are designed for streaming constant-bit rate (CBR) encoded videos and thus cannot benefit from the improved visual quality obtainable from variable-bit rate (VBR) encoding techniques. To tackle this challenge, this paper presents a turbo-slice-and-patch (TSP) algorithm to support VBR video streaming in a multicast VoD system. Results obtained from trace-driven simulation of $300 \mathrm{VBR}$ videos show that serving VBR videos with the TSP algorithm increases the average latency by only $9 \%$ compared to the CBR case with the same average video bit rate. Moreover, in 165 out of the 300 video titles, the TSP algorithm actually outperforms the CBR equivalent by shortening the latency by $0.04 \%-99 \%$. Given that we can achieve similar visual quality by encoding VBR video at half the average rate of CBR video, this TSP algorithm can potentially serve VBR videos with more consistent visual quality and with less resource compare to CBR-based video streaming systems.
\end{abstract}

Index Terms-Turbo-slice-and-patch (TSP), variable bit rate (VBR) video streaming, video multicast, video-on-demand (VoD).

\section{INTRODUCTION}

$\mathbf{I}$ $\mathrm{N}$ A true-video-on-demand ( $\mathrm{TVOD}$ ) system, the video server has to reserve a dedicated video channel for each user for the entire duration of the video session (e.g., two hours for a movie). Consequently, the server and network resources required increase linearly with the number of concurrent users to be supported. Although current PC servers are already very powerful and capable to serve up to hundreds of concurrent video streams, scaling up a system to thousands and even millions of concurrent video streams is still prohibitively expensive.

One promising solution to this scalability challenge is through the intelligent use of network multicast. Network multicast enables a server to send a few streams of video data for reception by a large number of clients, thereby significantly

Manuscript received November 3, 2003; revised May 27, 2005. This research was supported in part by Direct and Earmarked Grant CUHK 4328/02E from the Hong Kong Research Grant Council and in part by the Area of Excellence Scheme, established under the University Grants Council of the Hong Kong Special Administrative Region, China, under Project no. AoE/E-01/99. This paper was recommended by Associate Editor O. Al-Shaykh.

C.-W. Kong and J. Y. B. Lee are with the Department of Information Engineering, The Chinese University of Hong Kong, Hong Kong, SAR (e-mail: yblee@ie.cuhk.edu.hk).

M. Hamdi is with the Department of Computer Science, Hong Kong University of Science and Technology, Clear Water Bay, Kowloon, Hong Kong, SAR (e-mail: hamdi@cs.ust.hk).

V. O. K. Li is with the Department of Electrical and Electronic Engineering, The University of Hong Kong, Hong Kong, SAR (e-mail: vli@eee.hku.hk).

Digital Object Identifier 10.1109/TCSVT.2006.870021 reducing the amount of resources required. A number of pioneering studies have investigated such architectures, such as batching [2]-[4], patching [5]-[8], and periodic broadcasting [9]-[12].

A common assumption among these multicast VoD architectures is that the videos are constant bit rate (CBR) encoded. This significantly simplifies system design and analysis, and enables one to study the system performance independent of video encoding variations. Nevertheless, the visual quality of CBR video is not constant and tends to vary according to the video content. For example, complex video scenes with a lot of motion will typically result in lower visual quality than simple video scenes with little movement.

By contrast, videos encoded with constant-quality encoding algorithms will have more consistent visual quality, albeit at the expense of bit rate variations. However, a study by Tan et al. [14] has shown that VBR-encoded video can achieve visual quality similar to CBR-encoded video using only half the bit rate. This result suggests that VBR encoding is not only desirable for providing high-quality $\mathrm{VoD}$ services, but also has the potential to reduce resource requirements as well. The challenge is the complex resource allocation and scheduling problems resulting from the video bit rate variations.

This study addresses this challenge by investigating a new turbo-slice-and-patch (TSP) algorithm for serving VBR-encoded video streams in a metropolitan-scale video streaming service using network multicast. Unlike previous work on VBR video streaming that focused on unicast network transmission, the TSP algorithm investigated in this study employs network multicast to significantly increase the system's scalability to cope with the immense workload in a metropolitan scale streaming service. Results obtained from trace-driven simulation of $300 \mathrm{VBR}$ videos show that serving VBR videos with the TSP algorithm increases the average latency by only $9 \%$ compared to the CBR case with the same average video bit rate. Moreover, in 165 out of the 300 video titles, the TSP algorithm actually outperforms the CBR equivalent by shortening the latency by $0.04 \%-99 \%$. Given that we can achieve similar visual quality by encoding VBR video at half the average rate of CBR video, this TSP algorithm can potentially serve VBR videos with more consistent visual quality and with less resource compare to CBR-based video streaming systems.

The rest of the paper is organized as follows. Section II reviews some related work and compares them with this study. Section III reviews the multicast VoD architecture. Section IV presents two priority scheduling algorithms. Section V presents the slice-and-patch $(\mathrm{S} \& \mathrm{P})$ algorithm. Section VI presents a 
proof for continuous playback guarantee and derives the client buffer requirment. Section VII evaluates and compares the three algorithms using simulation results and Section VIII concludes the paper.

\section{BACKGROUND}

The problem of VBR video delivery in unicast VoD systems has been studied extensively. We review some of the more relevant previous work in Section II-A and compare them with this study in Section II-B.

\section{A. Previous Work}

One of the best known solutions for VBR video delivery is temporal smoothing [15]-[20]. Smoothing makes use of a client-side buffer to receive data in advance of playback. This work-ahead technique enables the server to transmit video data in a piecewise linear schedule that can be optimized to minimize rate variability [16], to minimize the number of rate changes [17], or to guarantee quality-of-service [19]. The schedule can be computed offline and with proper resource reservation, deterministic performance can be guaranteed. Interested readers are referred to Feng et al. [20] for a thorough comparison of various smoothing algorithms.

In another study by Lee and Yeom [], a data prefetch technique is proposed to improve video server performance in serving VBR videos. Unlike smoothing, where all video data are retrieved from the disk sequentially, data prefetching preloads video data corresponding to a video's bit rate peaks into the server's memory during system initialization. During operation, the server then only needs to retrieve the remaining video data from the disk to combine with the prefetched data for transmission to the clients. As the remaining video stream has a lower peak bit rate, disk utilization is increased. Their simulation results show that up to $81 \%$ more streams can be served using this prefetch technique. The tradeoffs are increased server buffer requirement and additional offline preprocessing of the video data.

A third approach proposed by Saparilla et al. [9] schedules video data transmission using a priority scheduler (join-the-shortest queue). In particular, the server schedules video data transmission according to the demand of data of each channel. A channel with the greatest demand of data (the clients listening to this channel are most likely to run out of data) will have the highest priority in the next round of transmission. However, while server efficiency is improved, this priority scheduler does not guarantee a client can receive all data in time. In particular, a channel will simply be skipped (i.e., not transmitted) if the data cannot be transmitted in time for playback. Their simulation results show that with their join-the-shortest queue priority scheduling and allowing the client to retrieve data from seven channels synchronously, the start-up latency can be limited to around 100 seconds with a loss probability of $10^{-6}$.

\section{B. Comparison}

Compared to the TSP algorithm, both temporal smoothing and the data prefetch techniques discussed previously are orthogonal and complementary. For temporal smoothing, a smoothed VBR video stream can be considered as just another VBR video stream, albeit one requiring additional client buffer for proper playback. For the data prefetch technique, the focus is on improving disk retrieval efficiency by intelligently preloading some video data into the server memory. Obviously, this technique does not affect the transmission schedule at all and can thus be integrated with any transmission scheduling algorithms including TSP.

Compared to the study by Saparilla et al. [9], TSP differs in two major ways. First, the TSP algorithm guarantees that no video data will be skipped, thus ensuring visual quality. Second, TSP is targeted at clients with limited access bandwidth (twice the average bit rate of the video). By contrast, the algorithm proposed by Saparilla et al. assumes the client have sufficient bandwidth to receive data from many channels simultaneously, which may not be practical for some applications.

In a previous work [1], we investigated an early version of the TSP algorithm, the S\&P, which shares some of the design principles of the TSP algorithm. There are, however, two important differences. First, in S\&P the VBR video is first smoothed using temporal smoothing (e.g., optimal smoothing [16]) before being subjected to the slicing operation. In TSP, we divide the video into two sections that are independently smoothed. Second, TSP has a different algorithm in phase 2 of the patching process (c.f. Section V-C) where video data are transmitted at the maximum client access bandwidth rather than the original video bit rate in S\&P (hence the name TSP versus S\&P [1]). Last but not least, we have conducted more extensive trace-driven simulation using 300 VBR video traces in this study (versus 50 in the previous study [1]) to evaluate the proposed algorithms in a much broader context to obtain more complete and reliable results.

\section{SySTEM ARCHITECTURE}

In this section, we briefly review the super-scalar VoD (SSVoD) architecture proposed by Lee and Lee [13]. SSVoD is designed for streaming CBR videos over a combination of static and dynamically-scheduled multicast transmission channels. Its primary advantages are the super-linear scalability achieved by multicast transmission, and the ability to support interactive playback control such as pause-resume and slow-motion playback without additional server resources.

\section{A. Transmission Scheduling}

The SSVoD architecture comprises a number of service nodes delivering video data over multicast channels to the clients. SSVoD achieves scalability and bandwidth efficiency by sending video data to a large number of clients using a few multicast channels. However, simple periodic multicast schemes such as those used in a near-video-on-demand (NVoD) system limit the time for which a client may start a new video session. Depending on the number of multicast channels allocated for a video title, this startup delay can range from a few minutes to tens of minutes. To tackle this initial delay problem, SSVoD employs patching to enable a client to start video playback at any time using a dynamic multicast channel 
until it can be merged back onto an existing multicast channel. The following sections present these techniques in more detail.

Each service node in the system streams video data using multiple multicast channels. Let $M$ be the number of video titles served by each service node and let $N$ be the total number of multicast channels available to a service node. For simplicity, we assume $N$ is divisible by $M$ and hence each video title is served by the same number of multicast channels, denoted by $N_{M}=N / M$. These multicast channels are then divided into $N_{S}$ static multicast channels and $N_{D}=N_{M}-N_{S}$ dynamic multicast channels. The video title is multicast repeatedly over all $N_{S}$ static multicast channels in a time-staggered manner as shown in Fig. 1. Specifically, adjacent channels are offset by

$$
T_{R}=\frac{L}{N_{S}}
$$

seconds, where $L$ is the length of the video in seconds. Transmissions are repeated continuously, i.e., restarted from the beginning of a video title every time transmission completes, regardless of the load of the server or how many users are active. These static multicast channels are used as the main channels for delivering video data to the clients. A client may start out with a dynamic multicast channel but it will shortly be merged back to one of these static multicast channels as explained in the next section.

\section{B. Admission Control}

To reduce the response time while still leveraging the bandwidth efficiency of multicast, SSVoD allocates a portion of the multicast channels and schedules them dynamically according to the request arrival pattern. A new user either waits for the next upcoming multicast transmission from a static multicast channel, or starts playback with a dynamic multicast channel.

Suppose a request arrives at time $t_{0}$, between the start time of the previous multicast cycle, denoted by $t_{m}$, and the start time of the next multicast cycle, denoted by $t_{m+1}$ (see Fig. 1). The new request will be assigned to wait for the next multicast cycle to start playback if the waiting time, denoted by $w_{i}$, is equal to or smaller than a predefined admission threshold $2 \delta$, i.e., $w_{i}=t_{m+1}-t_{0} \leq 2 \delta$. We call these requests statically admitted. This admission threshold is introduced to reduce the amount of load going to the dynamic multicast channels.

On the other hand, if the waiting time is longer than the threshold, then the client will request a dynamic multicast channel to begin playback (dynamically admitted), while simultaneously caching video data from the multicast channel with the multicast cycle started at time $t_{m}$. Note that the client may need to wait for a dynamic multicast channel to become available. If additional clients requesting the same video arrive during the wait, they will be batched and served by the same dynamic multicast channel once it becomes available. Eventually, the client playback will reach the point where the cached data began and then it can release the dynamic multicast channel and continue playback using data received from the static multicast channel. This integration of batching with patching significantly increases the system's efficiency at heavy loads.

Compared to TVoD systems, an SSVoD client must have the capability to receive two multicast channels concurrently and



Fig. 1. Patching process in the SSVoD system supporting CBR video.

have a local buffer to hold up to $T_{R}$ seconds of video data. Given a video bit rate of $3 \mathrm{Mbps}$ (e.g., high-quality MPEG-4 video), a total of 6 Mbps downstream bandwidth is required during the initial patching phase of the video session. For a 2 -hr movie served using 25 static multicast channels, the buffer requirement is $108 \mathrm{MB}$. This can easily be accommodated using a small harddisk at the client, and in the near future simply using memory as technology improves.

\section{Challenges in Supporting VBR-Encoded Video}

The SSVoD architecture is designed for CBR videos and thus problems will arise if stream VBR videos using the architecture. The first problem is in channel allocation. SSVoD partitions the server and network bandwidth into fixed-bandwidth network channels for allocation purpose. This allocation model is clearly undesirable for streaming VBR videos as it requires each channel to have sufficient bandwidth to accommodate the peak rate of the video, which is typically many times the average video bit rate.

To tackle this problem, we need to abandon the fixed-rate channel allocation model altogether and resort to allocating bandwidth according to the exact video bit rate profile. Specifically, instead of reserving half the channels for static multicast channels, we reserve half the server and network bandwidth for multicasting VBR streams in a time-staggered manner. Let the function $v(t)$ define the bit rate at which video data are being consumed $t$ seconds after playback has begun. To multicast a video title in $n$ independent time-staggered streams, the aggregate video bit rate of the ensemble, denoted by $V_{S}(t, n)$ will be given by

$$
V_{S}(t, n)=\sum_{i=0}^{n-1} v\left(\left(t \bmod \frac{L}{n}\right)+\frac{i L}{n}\right)
$$

Thus we can determine the maximum number of time-staggered VBR video streams that can fit within the system capacity from

$$
N_{S}=\max \left\{n \mid V_{S}(t, n) \leq 0.5 C, \forall t, n\right\}
$$

where $C$ is the total server and network bandwidth available.

The second problem is in the client access network where the client has an access network bandwidth equal to twice the video bit rate. While this is sufficient for receiving two CBR video streams, it may run into congestion when VBR video is streamed 
due to video bit rate variations. The use of temporal smoothing can alleviate this problem but cannot solve it completely without adding excessive start-up delay. In the next section, we address this problem by presenting two streaming algorithms based on priority scheduling.

\section{PRIORITY SCHEDULING}

The primary problem in streaming VBR video in SSVoD is that dynamically-admitted clients may not have sufficient access bandwidth to accommodate both the dynamic and the static multicast channel. For example, let $R_{V}$ be the average video bit rate, then the client has an access bandwidth of $2 R_{V}$. However, a VBR video of average bit rate $R_{V}$ will likely have bit rate peaks substantially higher than $R_{V}$ even after smoothing is applied (e.g., some of the videos in our collection have average bit rate $4 \mathrm{Mbps}$ but have peak bit rate exceeding $12 \mathrm{Mbps}$ ). It is easy to see that the access channel will become seriously congested whenever peaks from both dynamic channel and static channel overlap.

Obviously, we can increase the access network bandwidth to accommodate the overlapping bit rate peaks. However this trivial solution suffers from two limitations. First, the access network bandwidth is often limited by the access network technology employed. For example, if Ethernet is employed as the access network infrastructure, then the access bandwidth can never exceed $10 \mathrm{Mbps}$ (lower in practice due to frame/packet header overheads). Thus in this case the access network cannot even accommodate one single stream of the VBR video (which has peaks over $10 \mathrm{Mbps}$ ), let alone two streams. Second, the precise access bandwidth required is dependent on the particular video being streamed, thus rendering it impossible to fix the access network bandwidth during system design.

Therefore, we present in the following two priority-scheduling algorithms that can operate with a given access network bandwidth (say two times the average video bit rate) and yet are able to support the caching and patching operations in SSVoD.

\section{A. Static Channel Priority (SCP)}

In the SCP algorithm, we schedule the static channels to transmit at the original video bit rate. The dynamic channel will simply use the remaining access network bandwidth to transmit video data for patching so that the aggregate bit rate does not exceed the access bandwidth limit. Before streaming can start, we will process the video offline by collecting all the data above the bit rate $R_{\max }$ for $0 \leq \tau \leq L$ to form the prefetch block $P$, which is of bit rate $v_{p}(\tau)$ given by

$$
v_{p}(\tau)= \begin{cases}v_{0}(\tau)-R_{\max }, & \text { if } v_{0}(\tau)>R_{\max } \\ 0, & \text { otherwise }\end{cases}
$$

where $v_{0}(\tau)$ is the original video bit rate for any playback points $0 \leq \tau \leq L$. We can thus guarantee that the client's access bandwidth will be sufficient for caching data from the static channel after the dynamic channel is released. This block $P$ will be multicast periodically by a static channel at the bit rate $R_{\max }$. A client arriving at the system will first prefetch data from this channel for a period of $\left(1 / R_{\max }\right) \int_{0}^{L} v_{p}(t) d t$ seconds.
Assume the client finishes prefetching at time $t_{0}$ and the immediate previous multicast cycle begins at time $t_{m}$. The client will immediately begin caching video data from the static multicast channel starting from a playback point of $t_{0}-t_{m}$ and request a dynamic channel to stream the missed video data from playback point 0 to $t_{0}-t_{m}$. Then the amount of residual access bandwidth left for the dynamic channel at time $t$ is equal to $u(t)=R_{\max }-v\left(t-t_{m}\right)$ for $t \geq t_{1}$ where $v(\tau)=v_{0}(\tau)-v_{p}(\tau)$ for all $0 \leq \tau \leq L$.

As the client has already missed the first $t_{0}-t_{m}$ seconds of the video, a dynamic channel will be allocated to stream video data from the beginning of the video to the playback point $t_{0}-t_{m}$. The transmission duration, denoted by $d_{m}$, can then be obtained by solving the following equation:

$$
\int_{t_{1}}^{t_{1}+d_{m}} u(\tau) d \tau=\int_{t_{1}}^{t_{1}+\left(t_{0}-t_{m}\right)} v\left(\tau-t_{1}\right) d \tau .
$$

However, since the residual bandwidth available to the dynamic channel may not be sufficient to sustain continuous playback, the client may need to introduce an extra delay before playback can begin. Specifically, if the following inequality is satisfied:

$$
\int_{t_{1}}^{t} u(\tau) d \tau \geq \int_{t_{1}}^{t} v\left(\tau-t_{1}\right) d \tau, \text { for } t_{1} \leq t \leq\left(t_{1}+d_{m}\right)
$$

then it implies that the amount of video data received from the dynamic channel always exceeds the amount required for continuous playback. In this case the client can begin playback as soon as the dynamic channel becomes available. Otherwise, the client will have to delay playback by say $d_{s}$ seconds so that the continuity condition is satisfied:

$$
\begin{array}{r}
d_{s}=\min \left\{d \mid \int_{t_{1}}^{t} u(\tau) d \tau \geq \int_{t_{1}+d}^{t} v\left(\tau-t_{1}-d\right) d \tau\right. \\
\text { for } \left.\left(t_{1}+d\right) \leq t \leq\left(t_{1}+d_{m}\right)\right\} .
\end{array}
$$

As we will show in Section VII, $d_{s}$ can become very large for certain videos.

Next we derive the client buffer requirement for SCP. Specifically, the client will need to buffer video data from both the dynamic channel and the static channel. First, we derive the amount of data received at any time $t$. From the time the client has finished prefetching at time $t_{0}$, data will be cached from the static channel up to the end of the video section at time $\left(t_{m}+L\right)$. The amount of data received from the static channel at any time $t$ where $t_{0} \leq t \leq\left(t_{m}+L\right)$ is equal to $\int_{t_{0}}^{t} v\left(\tau-t_{m}\right) d \tau$. To simplify notations, we set $v(\tau)=0$ for all $\tau>L$ and $\tau<0$. From time $t_{1}$ to time $t_{1}+d_{m}$, the client will receive video data from the dynamic channel at the rate $u(t)$. Thus the accumulated amount of video data received by time $t$ is equal to $\int_{t_{1}}^{t} u(\tau) d \tau$. Note that $u(t)=0$ for all $t<t_{1}$ (i.e., before dynamic channel becomes available) and $t>\left(t_{1}+d_{m}\right)$ (i.e., patching is completed). 
Therefore, the total amount of data received at any time $t$ is simply given by

$$
\int_{0}^{L} v_{p}(\tau) d \tau+\int_{t_{0}}^{t} v\left(\tau-t_{m}\right) d \tau+\int_{t_{1}}^{t} u(\tau) d \tau .
$$

Now as the client begins playback from time $t_{1}+d_{s}$, the cumulated amount of data consumed by the time $t$ for $t_{1}+d_{s} \leq t \leq$ $\left(t_{1}+d_{s}+L\right)$ is then given by

$$
\begin{aligned}
\int_{t_{1}+d_{s}}^{t}\left[v\left(\tau-\left(t_{1}+d_{s}\right)\right)+v_{p}\left(\tau-\left(t_{1}+d_{s}\right)\right)\right] d \tau \\
=\int_{t_{1}+d_{s}}^{t} v\left(\tau-\left(t_{1}+d_{s}\right)\right) d \tau+\int_{0}^{t-\left(t_{1}+d_{s}\right)} v_{p}(\tau) d \tau .
\end{aligned}
$$

Finally, we can compute the amount of excess data received but not yet played back at any time $t$ from

$$
\begin{aligned}
& U_{\text {scp }}\left(t_{0}, t_{1}, t\right) \\
& =\left(\int_{0}^{L} v_{p}(\tau) d \tau+\int_{t_{0}}^{t} v\left(\tau-t_{m}\right) d \tau+\int_{t_{1}}^{t} u(\tau) d \tau\right) \\
& \quad-\left(\int_{t_{1}+d_{s}}^{t} v\left(\tau-\left(t_{1}+d_{s}\right)\right) d \tau+\int_{0}^{t-\left(t_{1}+d_{s}\right)} v_{p}(\tau) d \tau\right) \\
& =\int_{t-\left(t_{1}+d_{s}\right)}^{t} v_{p}(\tau) d \tau+\int_{t_{0}}^{t} v\left(\tau-t_{m}\right) d \tau \\
& \quad+\int_{t_{1}}^{t} u(\tau) d \tau-\int_{t_{1}+d_{s}}^{t} v\left(\tau-\left(t_{1}+d_{s}\right)\right) d \tau .
\end{aligned}
$$

The maximum of (10) thus determines the client buffer requirement

$$
\begin{aligned}
U_{\mathrm{scp}}^{\max }= & \max \left\{U_{\mathrm{scp}}\left(t_{0}, t_{1}, t\right), \forall t_{0}, t_{1}, t \mid t_{m} \leq t_{0} \leq\left(t_{m}+T_{r}\right),\right. \\
& \left.t_{0} \leq t_{1} \leq\left(t_{m}+T_{r}\right), t_{1} \leq t \leq\left(t_{1}+d_{s}+L\right)\right\} .
\end{aligned}
$$

\section{B. Dynamic Channel Priority (DCP)}

To avoid the startup delay in the previous SCP algorithm, we can alternatively give priority to the dynamic channel which streams video data starting from the beginning. Unlike the previous algorithm, the static channel cannot simply transmit video data using the leftover access bandwidth because the static channels are periodically multicast in a fixed schedule and bit rate profile to a large number of clients. Therefore, once a dynamic channel becomes available at time $t_{1}=t_{0}+w$, the server will transmit video data from the beginning of the video to the client at the maximum rate $R_{\max }$ until it catches up with the playback point, say $s$, currently being multicast by the static channel at time $t_{m}+s$. At that instant, the client can then release the dynamic channel and continue receiving data from the static channel for the rest of the session. Similar to the SCP algorithm, the video is processed offline to extract video data exceeding the client access bandwidth into a prefetch block $P$, which is then multicast periodically at the rate $R_{\max }$. The client will prefetch block $P$ before requesting for a dynamic channel to begin playback.
Unlike SCP however, the client in DCP can always begin playback once a dynamic channel is available. The client does not cache video data from a static channel until the dynamic channel catches up with the playback point currently being broadcast by the static channel. When the dynamic channel becomes available at time $t_{1}$ and releases at time $\left(t_{m}+s\right)$, the client would have received video data of size $R_{\max } \cdot\left(t_{m}+s-t_{1}\right)$, while missed video data from playback point 0 to $s$ of size given by $\int_{0}^{s} v(\tau) d \tau$. So, to determine this switchover point, we need to find $s$ that satisfies the following equation:

$$
R_{\max } \cdot\left(t_{m}+s-t_{1}\right)=\int_{0}^{s} v(\tau) d \tau, \text { where } t_{m}+s \geq t_{1} .
$$

In comparison, the dynamic channel in DCP will consume more resource than its SCP counterpart. In particular, the dynamic channel itself is streamed at the maximum access bit rate (i.e., $\left.R_{\max }\right)$. Also, the client cannot cache video data from the static channel while the dynamic channel is streaming, thus increasing the time it takes to catch up with the static channel. Both factors increase the dynamic channel's bandwidth consumption.

To determine the client buffer requirement, we first consider the amount of data received from the dynamic channel. At time $t_{1}$, the dynamic channel starts streaming data to the client at the rate $R_{\max }$ up to the time $\left(t_{m}+s\right)$ when the dynamic channel is released. The accumulated amount of data received from the dynamic channel by time $t$ where $t_{1} \leq t \leq\left(t_{m}+s\right)$ is given by

$$
r(t) \cdot\left(t-t_{1}\right)
$$

where $r(t)$ equals $R_{\max }$ for $t_{1} \leq t \leq\left(t_{m}+s\right)$ or 0 otherwise. After the dynamic channel finishes, i.e., time $t \geq\left(t_{m}+s\right)$, the client will cache data from the static channel and thus the accumulated amount of data received by time $t$ where $\left(t_{m}+\right.$ $s) \leq t \leq\left(t_{m}+L\right)$ is given by $\int_{t_{m}+s}^{t} v_{r}\left(\tau-t_{m}\right) d \tau$, where $v_{r}(\tau)=v(\tau)$ for $s \leq \tau \leq L$ or 0 otherwise because video data of playback point before $s$ will not be received from the static channel. Therefore, the total amount of data received by time $t$ where $t_{1} \leq t \leq\left(t_{m}+L\right)$ is then given by

$$
\int_{0}^{L} v_{p}(\tau) d \tau+r(t) \cdot\left(t-t_{1}\right)+\int_{t_{m}+s}^{t} v_{r}\left(\tau-t_{m}\right) d \tau .
$$

Now as the client begins playback at time $t_{1}$, the accumulated amount of data consumed by time $t$ is given by $\int_{t_{1}}^{t}\left[v\left(\tau-t_{1}\right)+\right.$ $\left.v_{p}\left(\tau-t_{1}\right)\right] d \tau$. Thus the excess amount of video data received but not yet played back at time $t$ is given by

$$
\begin{aligned}
& U_{\mathrm{dcp}}\left(t_{1}, t\right) \\
& =\left(\int_{0}^{L} v_{p}(\tau) d \tau+r(t) \cdot\left(t-t_{1}\right)+\int_{t_{m}+s}^{t} v_{r}\left(\tau-t_{m}\right) d \tau\right) \\
& \quad-\left(\int_{t_{1}}^{t} v\left(\tau-t_{1}\right) d \tau+\int_{0}^{t-t_{1}} v_{p}(\tau) d \tau\right) \\
& =\int_{t_{m}+s}^{t} v_{r}\left(\tau-t_{m}\right) d \tau+\int_{t_{1}}^{t}\left[r(t)-v\left(\tau-t_{1}\right)\right] d \tau \\
& \quad+\int_{t-t_{1}}^{L} v_{p}(\tau) d \tau .
\end{aligned}
$$


The maximum of (15) thus determines the client buffer requirement

$$
\begin{array}{r}
U_{\mathrm{dcp}}^{\max }=\max \left\{U_{\mathrm{dcp}}\left(t_{1}, t\right), \forall t_{1}, t \mid t_{m} \leq t_{1} \leq\left(t_{m}+T_{r}\right),\right. \\
\left.t_{1} \leq t \leq\left(t_{1}+d_{s}+L\right)\right\} .
\end{array}
$$

\section{TURBO-SLICE-AND-PATCH (TSP)}

The two priority scheduling algorithms presented in the previous section have their pros and cons. In this section, we present the TSP algorithm that combines the virtues of the SCP and the DCP algorithms. In TSP, we divide the video stream into three portions (i.e., slicing) and admit clients using a three-phase patching process (i.e., patching). The following sections present the algorithm in detail.

\section{A. Video Preprocessing}

Before a video is put online for streaming, two offline processing steps are performed: temporal smoothing and slicing of the video. First, we apply temporal smoothing [16] to reduce the video's peak bit rate. However, experiments show that temporal smoothing may also increase bandwidth consumption during the patching process. This is because temporal smoothing employs work-ahead to aggressively stream video data to the client as long as buffer allows. Consequently, this work-ahead mechanism will substantially increase the transmission rate of the video's initial portion, thus increasing the time to complete the patching process.

To tackle this problem, we divide the video into two segments and then perform temporal smoothing to these two segments independently. The first video segment comprises video data from the beginning to the playback point $T_{A}$ given by

$$
T_{A}=\left(\frac{R_{\max }}{R_{\max }-R_{\text {cut }}}\right) T_{R}
$$

where $T_{R}$ is the repeating interval for the static multicast channels. The physical meaning of $T_{A}$ is the latest possible playback point when the three-phase patching process will end. We will derive $T_{A}$ in Section V-C after we have presented the threephase patching process.

The rest of the video data then form the second video segment. This two-segment smoothing process can substantially reduce the initial transmission bit rate as the work-ahead algorithm will not transmit ahead of time video data beyond the playback point $T_{A}$. To simplify discussions, we will refer to the smoothed video bit rate simply as the video bit rate.

In the second step, we slice the smoothed video into three parts for transmission in three separate multicast channels. As depicted in Fig. 2, the video data stream is sliced at two bit rate thresholds: $R_{\text {cut }}$ and $\left(R_{\max }-R_{\text {cut }}\right)$, where $R_{\max }$ is the maximum access bandwidth of the client and $R_{\text {cut }}$ is a system parameter configurable from $R_{V}$ to $(2 / 3) R_{\max }$.

The first part, slice A, comprises two portions. The first portion includes video data exceeding the bit rate $R_{\text {cut }}$ (e.g., $\mathrm{A}_{1}$, $\mathrm{A}_{2}$, etc., in Fig. 2) from the beginning of the video until the playback point $T_{A}$ given by (17). The purpose of this slicing is to reduce the peak rate of the video stream to prevent congesting the client's access channel during patching. The second portion includes video data exceeding the client access bandwidth $R_{\max }$

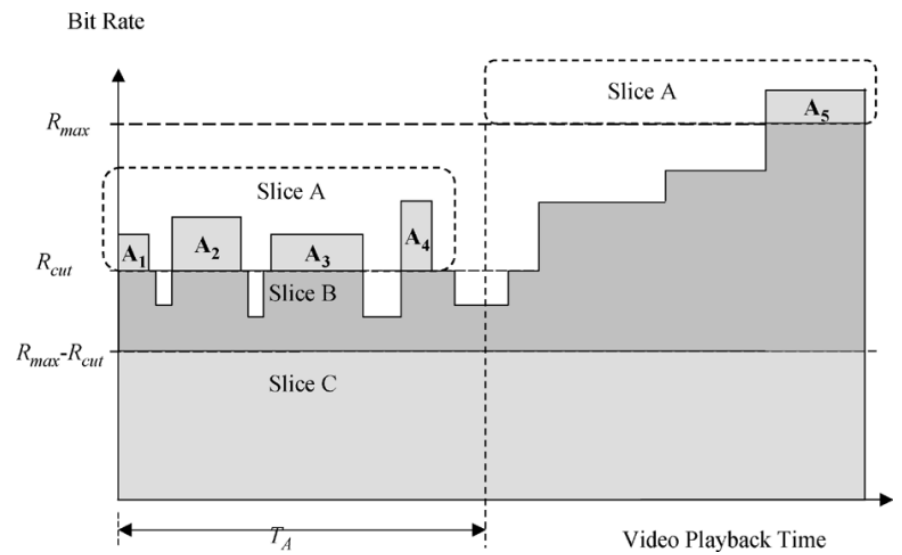

Fig. 2. Video slicing in the S\&P algorithm.

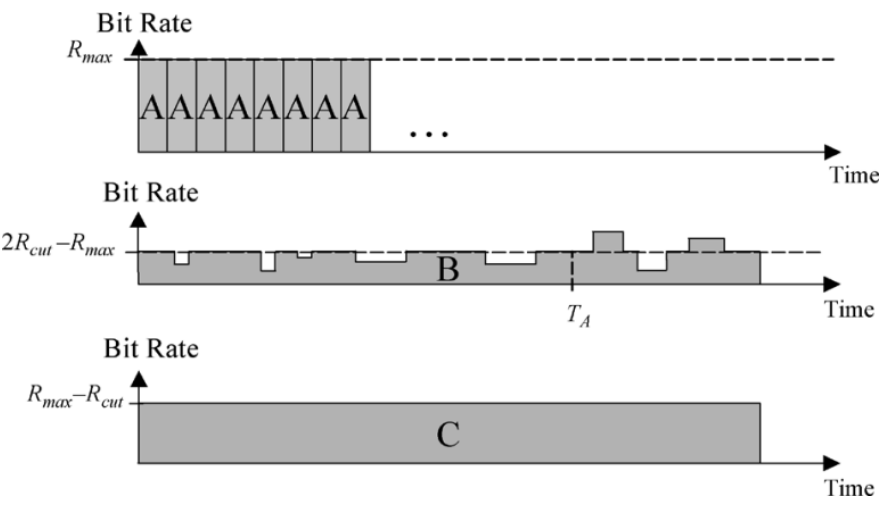

Fig. 3. Three types of multicast channels in the S\&P algorithm.

from the playback point $T_{A}$ to the end of the video. This portion is similar to the prefetch block in the SCP/DCP algorithms and the purpose is to keep the video streaming bit rate within the client access bandwidth limit. Let the size of this first video data block be $A \mathrm{Mb}$. It will be multicast repeatedly at a rate of $R_{\max }$ once every $d_{1}=A / R_{\max }$ seconds as shown in Fig. 3 .

The second part, slice B in Fig. 2, also comprises two portions. The first portion, covering the first $T_{A}$ seconds of the video, includes the video data that exceed the bit rate $\left(R_{\max }-\right.$ $R_{\text {cut }}$ ) but excludes those already in slice A. The second portion, covering from playback point $T_{A}$ until the end of the video, comprises all video data that exceeds the bit rate $\left(R_{\max }-R_{\text {cut }}\right)$ except those already in slice A. This slice will be multicast repeatedly over a separate multicast channel following the actual video bit rate (as opposed to the constant transmission rate for slice A) as shown in Fig. 3.

Lastly, the third part, slice $\mathrm{C}$ in Fig. 2, comprises the rest of the video data not included in slice $\mathrm{A}$ and slice $\mathrm{B}$. This slice will be multicast repeatedly over a third multicast channel following the actual video bit rate as shown in Fig. 3.

\section{B. Bandwidth Allocation}

Let $B_{\max }$ be the total server (or network, whichever is smaller) bandwidth allocated for a video of average bit rate $R_{V}$ bps and length $L$ seconds. First, a bandwidth of $R_{\max }$ will be allocated to multicast slice $\mathrm{A}$. Then the remaining bandwidth will be divided equally between the static multicast channels and dynamic multicast channels (c.f. Section VII-F). 


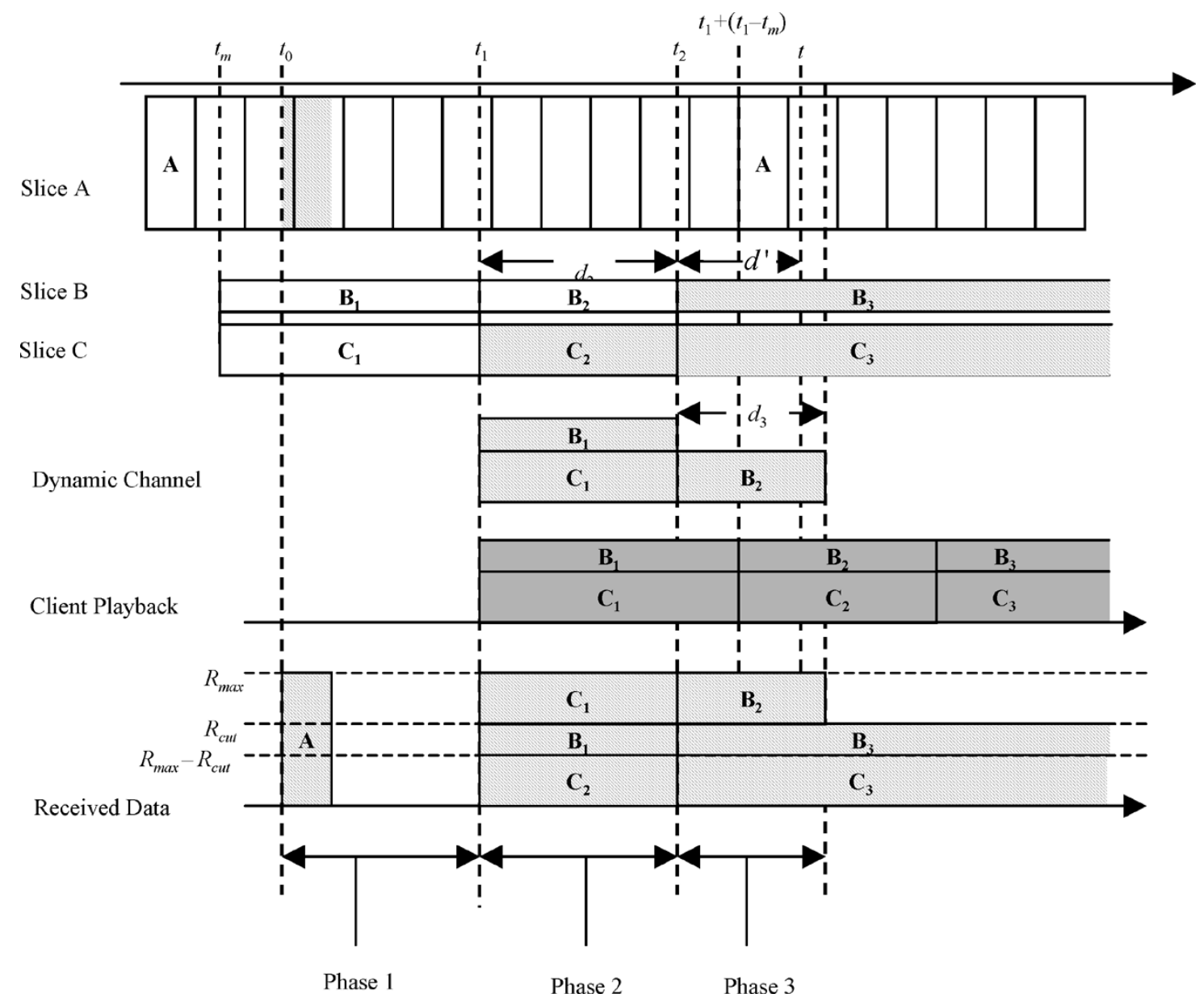

Fig. 4. Three-phase patching process in the S\&P algorithm.

There are two types of static multicast channels, transmitting slice $B$ and slice $C$, respectively. As the numbers of these channels are equal, we will refer to a pair of such channels as a static multicast channel. Unlike in the case of CBR videos, a static multicast channel in TSP does not occupy a constant amount of bandwidth. Therefore, offline numerical procedures are used to compute the maximum number of static multicast channels that can fit within the bandwidth limit $\left(B_{\max }-R_{\max }\right) / 2$. The remaining bandwidth will be used by the dynamic channels to patch newly admitted users and then merge them to one of the static multicast channels. Once the merging is completed, the user will not incur any additional load to the server for the rest of the video streaming session.

\section{Three-Phase Patching}

A new client goes through a three-phase patching process to begin a new video streaming session. Let the client arrive at time $t_{0}$. It immediately enters phase 1 by caching slice $\mathrm{A}$ at the maximum rate $R_{\max }$ for a duration of $d_{1}$ seconds as shown in Fig. 4 . Next, the client will request and wait for a dynamic channel to begin phase 2 . Once a dynamic channel becomes available at time $t_{1}$, the client begins receiving and playing back video data blocks $\left\{B_{1}, C_{1}\right\}$ while simultaneously caching block $C_{2}$ into a local buffer. The dynamic channel sustains video playback by streaming the missed content (i.e., blocks $\left\{B_{1}, C_{1}\right\}$ ) to the client at the bit rate $R_{\text {cut }}$. Thus the transmission duration in phase 2, denoted by $d_{2}$, is given by

$$
\begin{aligned}
d_{2} & =\frac{\int_{0}^{t_{1}-t_{m}} v_{b}(\tau) d \tau+\int_{0}^{t_{1}-t_{m}} v_{c}(\tau) d \tau}{R_{\text {cut }}} \\
= & \frac{\int_{0}^{t_{1}-t_{m}}\left[v_{b}(\tau)+v_{c}(\tau)\right] d \tau}{R_{\text {cut }}}
\end{aligned}
$$

where $v_{b}(\tau)$ and $v_{c}(\tau)$ are the transmission rates of slice B and slice $\mathrm{C}$ at playback point $\tau$. Note that for $\tau \leq T_{A}, v_{b}(\tau)+v_{c}(\tau)$ is bounded by $R_{\text {cut }}$ according to the slicing procedure described in Section V-A. Due to the rate varying nature of the video, it is possible that the combined bit rate $v_{b}(\tau)+v_{c}(\tau)$ is lower than $R_{\text {cut }}$ for some playback points. Thus, the transmission duration, denoted by $d_{2}$, cannot be larger than the length of video to be patched $\left(t_{1}-t_{m}\right)$

$$
d_{2} \leq\left(t_{1}-t_{m}\right) .
$$

By the end of phase 2, the client will have already cached block $C_{2}$ and completed playback of blocks $\left\{B_{1}, C_{1}\right\}$. However, due to the limited client access bandwidth, the client cannot cache block $B_{2}$ and thus in phase 3 the server will use the dynamic 
channel to stream block $B_{2}$ at the bit rate $\left(R_{\max }-R_{\text {cut }}\right)$ to sustain continuous video playback. Concurrently, the client continues to cache data (e.g., $B_{3}, C_{3}$ ) from the static channel for the rest of the video session. The duration of phase 3 , denoted by $d_{3}$, is given by

$$
d_{3}=\frac{1}{R_{\max }-R_{\mathrm{cut}}} \int_{t_{1}-t_{m}}^{t_{1}-t_{m}+d_{2}} v_{b}(\tau) d \tau
$$

where the numerator is the size of block $B_{2}$ and the denominator is the transmission rate. To deduce the upper bound for the transmission duration $d_{3}$, we would first show that the bit rate of slice $\mathrm{B}$ in the first $T_{A}$ seconds is actually bounded by $\left(R_{\max }-R_{\text {cut }}\right)$ in the following lemma.

Lemma 1: If $R_{\text {cut }} \leq(2 / 3) R_{\max }$, then $v_{b}(\tau) \leq\left(R_{\max }-\right.$ $R_{\text {cut }}$ ) for all $\tau \leq T_{A}$.

Proof: Firstly, $\left(R_{\max }-R_{\text {cut }}\right) \geq\left(R_{\max }-\left(2 R_{\max } / 3\right)\right)=$ $\left(R_{\max } / 3\right)$. Secondly, according to the slicing procedure, the bit rate of slice $\mathrm{B}$ is bounded by $R_{\text {cut }}-\left(R_{\text {max }}-R_{\text {cut }}\right)$ (Please refer to Fig. 2 and Fig. 3). Thus

$$
\begin{aligned}
v_{b}(\tau) & \leq R_{\text {cut }}-\left(R_{\text {cut }}-R_{\max }\right)=2 R_{\text {cut }}-R_{\max } \\
& \leq 2\left(\frac{2 R_{\max }}{3}\right)-R_{\max }=\frac{R_{\max }}{3}
\end{aligned}
$$

Thus, Lemma 1 follows.

From (20), we can now deduce the upper bound for the transmission duration $d_{3}$

$$
\begin{aligned}
d_{3}= & \frac{\int_{t_{1}-t_{m}}^{t_{1}-t_{m}+d_{2}} v_{b}(\tau) d \tau}{R_{\max }-R_{\text {cut }}} \\
\leq & \frac{\int_{1}-t_{m}}{t_{1}-t_{m}+d_{2}}\left(R_{\max }-R_{\text {cut }}\right) d \tau \\
= & d_{2} .
\end{aligned}
$$

With the three-phase patching process defined, we can proceed to derive $T_{A}$ used earlier in Section V-A.

Recall that (c.f. Section V-A) preprocessing of the video depends on the duration of the three-phase patching process, which in turns depends on the client arrival time. We first define $D\left(t_{1}\right)$ to be the length of time from $t_{m}$, i.e., the start of the multicast cycle, to the end of phase 3 given that the dynamic channel is available at time $t_{1}$. This time interval comprises three parts. The first part is the time from $t_{m}$ to the time the dynamic channel becomes available, having a length of $\left(t_{1}-t_{m}\right)$ seconds. The second part is the transmission duration of phase 2 as given by (18). The last part is the duration of phase 3 as given by (20). Thus, we can express $D\left(t_{1}\right)$ as

$$
D\left(t_{1}\right)=\left(t_{1}-t_{m}\right)+d_{2}+d_{3} .
$$

To determine the maximum duration of this interval, i.e., $T_{A}$, we first note that $\left(t_{1}-t_{m}\right)$ is upper-bounded by $T_{r}$ because it is the maximum time to the next multicast cycle. Thus if the dynamic channel is not available before then, the client can simply receive video data from the new multicast cycle to begin video playback. For the length of phase 2, we note that the combined bit rate $v_{b}(t)+v_{c}(t)$ cannot exceed $R_{\text {cut }}$ due to the slicing procedure. Thus, the maximum length of phase 2 can be computed from

$$
\begin{aligned}
d_{2}= & \frac{\int_{0}^{t_{1}-t_{m}}\left(v_{b}(t)+v_{c}(t)\right) d \tau}{R_{\text {cut }}} \\
\leq & \frac{\int_{0}^{t_{1}-t_{m}} R_{\text {cut }} d \tau}{R_{\text {cut }}} \\
= & t_{1}-t_{m} \leq T_{r} .
\end{aligned}
$$

Similarly, the maximum length of phase 3 is equal to the maximum size of segment $B_{2}$ (see Fig. 4) divided by the transmission rate $\left(R_{\max }-R_{\text {cut }}\right)$

$$
\begin{aligned}
d_{3}= & \frac{\int_{t_{1}-t_{m}}^{t_{1}-t_{m}+d_{2}} v_{b}(\tau) d \tau}{R_{\max }-R_{\text {cut }}} \\
\leq & \frac{\int_{t_{1}-t_{m}}^{t_{1}-t_{m}+d_{2}}\left(2 R_{\text {cut }}-R_{\max }\right) d \tau}{R_{\max }-R_{\text {cut }}} \\
= & \frac{\left(2 R_{\text {cut }}-R_{\text {max }}\right) \cdot d_{2}}{R_{\max }-R_{\text {cut }}} \\
\leq & \frac{\left(2 R_{\text {cut }}-R_{\text {max }}\right)}{R_{\text {max }}-R_{\text {cut }}} T_{r}
\end{aligned}
$$

due to (22). Finally, substituting (24) and (25) into the righthand side of (23) gives the desired result

$$
\begin{aligned}
D\left(t_{1}\right) & =\left(t_{1}-t_{m}\right)+d_{2}+d_{3} \\
& \leq T_{r}+T_{r}+\frac{2 R_{\text {cut }}-R_{\text {max }}}{R_{\text {max }}-R_{\text {cut }}} T_{r} \\
& =\left(\frac{2\left(R_{\max }-R_{\text {cut }}\right)}{R_{\max }-R_{\text {cut }}}+\frac{2 R_{\text {cut }}-R_{\max }}{R_{\max }-R_{\text {cut }}}\right) T_{r} \\
& =\frac{R_{\max }}{R_{\max }-R_{\text {cut }}} T_{r}=T_{A} .
\end{aligned}
$$

\section{Client Buffer Requirement}

In this section, we derive the maximum client buffer size needed under the TSP algorithm by considering the buffer required during the three phases of patching. First, in phase 1 the client prefetches slice $\mathrm{A}$ and thus the amount of buffer required is simply equal to the size of slice A, i.e., $\int_{0}^{T_{A}} v_{a}(\tau) d \tau$.

Phase 2 begins at time $t_{1}$ when the dynamic channel is available and finishes at time $t_{2}=t_{1}+d_{2}$. The client in this phase receives two streams of video data, one from the dynamic channel at the rate $R_{\max }$ and the other from the static channel multicasting slice $C$. Thus, the total amount of data received at any time during phase 2 (i.e., $t_{1} \leq t \leq t_{2}$ ) is given by

$$
\int_{0}^{T_{A}} v_{a}(\tau) d \tau+\int_{t_{1}}^{t} R_{\max } d \tau+\int_{t_{1}}^{t} v_{c}(\tau) d \tau .
$$

Phase 3 begins at time $t_{2}$ and finishes at time $t_{3}=t_{1}+d_{2}+d_{3}$. In this phase, the dynamic channel streams $B_{2}$ (see Fig. 4 ) to the 
client at the rate $\left(R_{\max }-R_{\text {cut }}\right)$. Concurrently, the client also caches data from the static channels multicasting slice $\mathrm{B}$ and slice $\mathrm{C}$. Thus, the total amount of data received at any time $t$ during phase 3 (i.e., $t_{2} \leq t \leq t_{3}$ ) is the sum of the total amount of data received up to time $t_{2}$ given by (27) plus the amount received during this phase up to time $t$, giving a total of

$$
\begin{aligned}
& \int_{0}^{T_{A}} v_{a}(\tau) d \tau+\int_{t_{1}}^{t_{2}} R_{\max } d \tau+\int_{t_{1}}^{t_{2}} v_{c}(\tau) d \tau \\
& +\int_{t_{2}}^{t}\left(R_{\max }-R_{\text {cut }}\right) d \tau+\int_{t_{2}}^{t}\left(v_{b}(\tau)+v_{c}(\tau)\right) d \tau \\
& =\int_{0}^{T_{A}} v_{a}(\tau) d \tau+\int_{t_{1}}^{t} R_{\max } d \tau+\int_{t_{1}}^{t} v_{c}(\tau) d \tau \\
& \quad+\int_{t_{2}}^{t} v_{b}(\tau) d \tau-\int_{t_{2}}^{t} R_{\text {cut }} d \tau .
\end{aligned}
$$

After phase 3 completes, i.e., at time $t_{3}$, the dynamic channel will be released and the client will continue caching both slice $\mathrm{B}$ and slice $\mathrm{C}$ from the static channel. So, the total amount of data received at any time $t$ where $t_{3} \leq t \leq t_{m}+L$ is simply the sum of the total amount of data received up to time $t_{3}$ given by (28) and that received after time $t_{3}$ giving a total amount of

$$
\begin{aligned}
& \int_{0}^{T_{A}} v_{a}(\tau) d \tau+\int_{t_{1}}^{t_{3}} R_{\max } d \tau+\int_{t_{1}}^{t_{3}} v_{c}(\tau) d \tau \\
& +\int_{t_{2}}^{t_{3}} v_{b}(\tau) d \tau-\int_{t_{2}}^{t_{3}} R_{\mathrm{cut}} d \tau+\int_{t_{3}}^{t}\left(v_{b}(\tau)+v_{c}(\tau)\right) d \tau \\
& =\int_{0}^{T_{A}} v_{a}(\tau) d \tau+\int_{t_{1}}^{t_{3}} R_{\max } d \tau+\int_{t_{1}}^{t} v_{c}(\tau) d \tau \\
& +\int_{t_{2}}^{t} v_{b}(\tau) d \tau-\int_{t_{2}}^{t_{3}} R_{\mathrm{cut}} d \tau
\end{aligned}
$$

To simplify notations, let

$$
r(t)= \begin{cases}R_{\max }, & \text { if } t_{1} \leq t \leq t_{2} \\ R_{\max }-R_{\text {cut }}, & \text { if } t_{2} \leq t \leq t_{3} \\ 0, & \text { otherwise }\end{cases}
$$

and

$$
v_{r}(t)= \begin{cases}v_{c}\left(t-t_{m}\right), & \text { if } t_{1} \leq t \leq t_{2} \\ v_{b}\left(t-t_{m}\right)+v_{c}\left(t-t_{m}\right), & \text { if } t_{2} \leq t \leq t_{m}+L \\ 0, & \text { otherwise }\end{cases}
$$

Then the sum of (27)-(29), i.e., the amount of data received by the client at any time $t$ where $t_{1} \leq t \leq t_{m}+L$ can be expressed as

$$
\int_{0}^{T_{A}} v_{a}(\tau) d \tau+\int_{t_{1}}^{t} r(t) d \tau+\int_{t_{1}}^{t} v_{r}(\tau) d \tau .
$$

Now as playback starts at $t_{1}$, the accumulated amount of data consumed by time $t$ is given by $\int_{0}^{t-t_{1}} v(\tau) d \tau$. Thus the excess amount of video data received but not yet played back at time $t$ where $t_{1} \leq t \leq t_{m}+L$ is given by

$U_{\mathrm{tsp}}\left(t_{1}, t\right)=\int_{0}^{T_{A}} v_{a}(\tau) d \tau+\int_{t_{1}}^{t} r(\tau) d \tau+\int_{t_{1}}^{t} v_{r}(\tau) d \tau-\int_{0}^{t-t_{1}} v(\tau) d \tau$

and the maximum of (33) thus determines the client buffer requirement

$$
\begin{array}{r}
U_{\mathrm{tsp}}^{\max }=\max \left\{U_{\mathrm{tsp}}\left(t_{1}, t\right), \forall t_{1}, t \mid t_{m} \leq t_{1} \leq\left(t_{m}+T_{r}\right),\right. \\
\left.t_{1} \leq t \leq\left(t_{1}+L\right)\right\} .
\end{array}
$$

\section{PlayBaCK CONTINUITY}

In the three-phase patching process, the client does not always receive video data according to the playback sequence. Consider the example in Fig. 4, the client receives in phase 2 video segments $B_{1}, C_{1}$, and $C_{2}$ simultaneously but $C_{2}$ is not played back until phase 3 . Consequently, to guarantee continuous video playback, it is not sufficient to just ensure the reception data rate is not lower than the video playback bit rate. In the following, we investigate this playback continuity issue and present a proof that TSP can indeed guarantee playback continuity for the entire video duration.

Let $v(\tau)$ be the playback bit rate of the video at playback point $\tau$ where $0 \leq \tau \leq L$. Let $v(\tau)=v_{a}(\tau)+v_{b}(\tau)+v_{c}(\tau)$ for $0 \leq$ $\tau \leq L$ where $v_{a}(\tau), v_{b}(\tau)$ and $v_{c}(\tau)$ are the playback bit rate of slice $\mathrm{A}$, slice $\mathrm{B}$ and slice $\mathrm{C}$ at playback point $\tau$, respectively. Let $c(t)$ be the total amount of continuous video data received by the client at time $t$. Assume the previous multicast of the static channel begins at time $t_{m}$ and the client starts playback at $t_{1}$ as shown in Fig. 4. Then to guarantee playback continuity we need to ensure that the amount of continuous video data received must always be larger than the amount required for continuous playback, or mathematically we need to establish that

$$
c(t) \geq \int_{t_{1}}^{t} v\left(\tau-t_{1}\right) d \tau \text { for } t_{1} \leq t \leq\left(L+t_{1}\right) .
$$

Note that as playback does not begin until phase 1 is completed, playback continuity is not affected by phase 1 . To derive $c(t)$ for the rest of the video session, we consider phase 2 and phase 3 in turn.

We first denote the amount of continuous data received up to time $t$ to be $c_{a}(t), c_{b}(t)$ and $c_{c}(t)$ for slice $\mathrm{A}$, slice $\mathrm{B}$, and slice $\mathrm{C}$, respectively. Since the client has already received the whole slice A after phase 1 is completed, $c_{a}(t)=\int_{0}^{t-t_{1}} v_{a}(\tau) d \tau$ at any time $t$ for $t_{1} \leq t \leq L+t_{1}$.

Phase 2 begins at time $t_{1}$ when a dynamic channel becomes available and ends at time $t_{2}=t_{1}+d_{2}$ when the dynamic channel has streamed all the missed data to the client. The following theorem proves the playback continuity during phase 2 .

Theorem 1: Video playback is continuous in phase 2, i.e., $c(t) \geq \int_{t_{1}}^{t} v\left(\tau-t_{1}\right) d \tau$, for $t_{1} \leq t \leq t_{2}$.

Proof: Consider $c_{b}(t)$ and $c_{c}(t)$. Since the dynamic channel streams blocks $\left\{B_{1}, C_{1}\right\}$ to the client continuously 
at the rate $R_{\text {cut }} \geq v_{b}(\tau)+v_{c}(\tau)$, the amount of video data transmitted by the dynamic channel at time $t, t \geq t_{1}$, can be computed from

$$
\begin{aligned}
\left(t-t_{1}\right) R_{\text {cut }} & =\int_{t_{1}}^{t} R_{\text {cut }} d \tau=\int_{0}^{t-t_{1}} R_{\text {cut }} d \tau \\
& \geq \int_{0}^{t-t_{1}}\left[v_{b}(\tau)+v_{c}(\tau)\right] d \tau .
\end{aligned}
$$

Next, we note that the dynamic channel streams slice B and slice $\mathrm{C}$ in a continuous playback sequence. Thus (36) also gives the amount of continuous video data received by the client

$$
\begin{aligned}
c_{b}(t)+c_{c}(t) & =\left(t-t_{1}\right) R_{\text {cut }} \geq \int_{0}^{t-t_{1}}\left[v_{b}(\tau)+v_{c}(\tau)\right] d \tau \\
& =\int_{t_{1}}^{t}\left[v_{b}\left(\tau-t_{1}\right)+v_{c}\left(\tau-t_{1}\right)\right] d \tau .
\end{aligned}
$$

Together with slice A already received, we can compute the total amount of continuous data received at any time $t$ during phase 2 from

$$
\begin{aligned}
c(t) & =c_{a}(t)+c_{b}(t)+c_{c}(t) \\
& =\int_{0}^{t-t_{1}} v_{a}(\tau) d \tau+c_{b}(t)+c_{c}(t) \\
& \geq \int_{0}^{t-t_{1}} v_{a}(\tau) d \tau+\int_{t_{1}}^{t}\left[v_{b}\left(\tau-t_{1}\right)+v_{c}\left(\tau-t_{1}\right)\right] d \tau \\
& =\int_{t_{1}}^{t} v_{a}\left(\tau-t_{1}\right) d \tau+\int_{t_{1}}^{t}\left[v_{b}\left(\tau-t_{1}\right)+v_{c}\left(\tau-t_{1}\right)\right] d \tau \\
& =\int_{t_{1}}^{t}\left[v_{a}\left(\tau-t_{1}\right)+v_{b}\left(\tau-t_{1}\right)+v_{c}\left(\tau-t_{1}\right)\right] d \tau \\
& =\int_{t_{1}}^{t} v\left(\tau-t_{1}\right) d \tau
\end{aligned}
$$

which shows that playback is always continuous during phase 2 .

Before we proceed to the next theorem, we first derive the latest possible end time of phase 2. From (19), $d_{2} \leq\left(t_{1}-t_{m}\right)$ because the dynamic channel may transmit slice $\mathrm{B}$ and slice $\mathrm{C}$ at a bit rate higher than the playback rate. Therefore, if we express $t_{2}$ in terms of $t_{1}$ and $d_{2}$, we can deduce the upper bound of $t_{2}$

$$
\begin{aligned}
t_{2} & =t_{1}+d_{2} \\
& \leq t_{1}+\left(t_{1}-t_{m}\right)
\end{aligned}
$$

In the next theorem, we prove that from $t_{2}$ up to $t_{1}+\left(t_{1}-t_{m}\right)$ within phase 3 , playback is also continuous.

Theorem 2: Playback is continuous, i.e., $c(t) \geq \int_{t_{1}}^{t} v(\tau-$ $\left.t_{1}\right) d \tau$, for $t_{2} \leq t \leq t_{1}+\left(t_{1}-t_{m}\right)$.

Proof: For $t \geq t_{2}$, the client will have received the video blocks $B_{1}, C_{1}$, and $C_{2}$, and is receiving $B_{2}$. Given that the sizes of $B_{1}$ and $C_{1}$ equal $\int_{0}^{t_{1}-t_{m}} v_{b}(\tau) d \tau$ and $\int_{0}^{t_{1}-t_{m}} v_{c}(\tau) d \tau$, respectively, we can obtain the following inequality:

$$
c_{b}(t)+c_{c}(t) \geq \int_{0}^{t_{1}-t_{m}}\left[v_{b}(\tau)+v_{c}(\tau)\right] d \tau .
$$

Therefore, we can compute the total amount of continuous video data received at time $t$ from

$$
\begin{aligned}
c(t)= & c_{a}(t)+c_{b}(t)+c_{c}(t) \\
\geq & \int_{0}^{t-t_{1}} v_{a}(\tau) d \tau+\int_{0}^{t_{1}-t_{m}}\left[v_{b}(\tau)+v_{c}(\tau)\right] d \tau \\
= & \int_{t_{1}}^{t} v_{a}\left(\tau-t_{1}\right) d \tau+\int_{0}^{t-t_{1}}\left[v_{b}(\tau)+v_{c}(\tau)\right] d \tau \\
& +\int_{t-t_{1}}^{t_{1}-t_{m}}\left[v_{b}(\tau)+v_{c}(\tau)\right] d \tau \quad \because t \leq t_{1}+t_{1}-t_{m} \\
\geq & \int_{t_{1}}^{t} v_{a}\left(\tau-t_{1}\right) d \tau+\int_{0}^{t-t_{1}}\left[v_{b}(\tau)+v_{c}(\tau)\right] d \tau \\
= & \int_{t_{1}}^{t} v_{a}\left(\tau-t_{1}\right) d \tau+\int_{t_{1}}^{t}\left[v_{b}\left(\tau-t_{1}\right)+v_{c}\left(\tau-t_{1}\right)\right] d \tau \\
= & \int_{t_{1}}^{t}\left[v_{a}\left(\tau-t_{1}\right)+v_{b}\left(\tau-t_{1}\right)+v_{c}\left(\tau-t_{1}\right)\right] d \tau \\
= & \int_{t_{1}}^{t} v\left(\tau-t_{1}\right) d t
\end{aligned}
$$

which shows that playback is continuous for $t_{2} \leq t \leq t_{1}+\left(t_{1}-\right.$ $\left.t_{m}\right)$.

Intuitively, Theorem 2 considers the duration after phase 2 when the client simply plays back the excess video data transmitted in phase 2 . The client accumulates excess video data because the dynamic channel in phase 2 may transmit slice $B_{1}$ and $C_{1}$ at a rate higher than the playback rate.

After phase 2, the client enters phase 3 of a duration denoted by $d_{3}$. In the following, we prove that playback is also continuous during phase 3.

Theorem 3: Video playback is continuous in phase 3, i.e., $c(t) \geq \int_{t_{1}}^{t} v\left(\tau-t_{1}\right) d \tau$, for $t_{2} \leq t \leq t_{2}+d_{3}$.

Proof: We consider two possible cases depending on the total duration of phase 2 and phase 3 .

Case I: $d_{2}+d_{3} \leq\left(t_{1}-t_{m}\right)$

In this case, $t_{2}+d_{3}=\left[\left(t_{1}+d_{2}\right)+d_{3}\right] \leq\left[t_{1}+\left(t_{1}-t_{m}\right)\right]$, which implies that phase 3 ends before the time $t_{1}+\left(t_{1}-t_{m}\right)$. As Theorem 2 has already established playback continuity for $t_{2} \leq t \leq t_{1}+\left(t_{1}-t_{m}\right)$, playback in phase 3 must also be continuous as well.

Case II: $d_{2}+d_{3} \geq\left(t_{1}-t_{m}\right)$

From Theorem 2, playback is continuous up to $t=t_{1}+$ $\left(t_{1}-t_{m}\right)$. Thus, in the following, we only need to consider the duration $\left[t_{1}+\left(t_{1}-t_{m}\right)\right] \leq t \leq\left[t_{2}+d_{3}\right]$ (see Fig. 4). 
During this duration, the dynamic channel is streaming block $B_{2}$ at a rate of $\left(R_{\max }-R_{\text {cut }}\right)$. Now, according to Lemma 1 , the playback rate of block $B_{2}$ must be lower than the dynamic channel's transmission rate, i.e., $v_{b}(\tau) \leq\left(R_{\max }-R_{\text {cut }}\right)$. Thus after a duration of $d^{\prime}=t-t_{2}$ from the start of phase 3 , the amount of video data streamed by the dynamic channel is equal to

$$
\int_{t_{2}}^{t_{2}+d^{\prime}}\left(R_{\max }-R_{\text {cut }}\right) d \tau \geq \int_{t_{1}-t_{m}}^{t_{1}-t_{m}+d^{\prime}} v_{b}(\tau) d \tau .
$$

The continuous data of slice B at time $t$ contains the whole video block $B_{1}$ and the part of block $B_{2}$ transmitted by the dynamic channel. Since the data are streamed in-order, the amount of continuous video data received $d^{\prime}$ seconds after phase 2 is given by

$$
\begin{aligned}
c_{b}(t) & =\int_{0}^{t_{1}-t_{m}} v_{b}(\tau) d \tau+\int_{t_{2}}^{t_{2}+d^{\prime}}\left(R_{\max }-R_{\text {cut }}\right) d \tau \\
& \geq \int_{0}^{t_{1}-t_{m}} v_{b}(\tau) d \tau+\int_{t_{1}-t_{m}}^{t_{1}-t_{m}+d^{\prime}} v_{b}(\tau) d \tau \\
& =\int_{0}^{t_{1}-t_{m}+d^{\prime}} v_{b}(\tau) d \tau .
\end{aligned}
$$

For slice $\mathrm{C}$, the continuous video data at time $t$ contains the whole video block $C_{1}$ streamed by the dynamic channel during phase 2, the whole video block $C_{2}$ cached from the static channel during phase 2 , and the portion of block $C_{3}$ transmitted by the dynamic channel. Since the video data is streamed in-order, the total amount of continuous data received $d^{\prime}$ seconds after phase 2 is given by

$$
\begin{aligned}
c_{c}(t) & =\int_{0}^{t_{1}-t_{m}} v_{c}(\tau) d \tau+\int_{t_{1}-t_{m}}^{t_{1}-t_{m}+d_{2}} v_{c}(\tau) d \tau+\int_{t_{2}}^{t_{2}+d^{\prime}} v_{c}(\tau) d \tau \\
& \geq \int_{0}^{t_{1}-t_{m}+d_{2}} v_{c}(\tau) d \tau .
\end{aligned}
$$

Therefore, the total amount of continuous data received by the client at time $t$ is given by

$$
\begin{aligned}
c(t)= & c_{a}(t)+c_{b}(t)+c_{c}(t) \\
\geq & \int_{0}^{t-t_{1}} v_{a}(\tau) d \tau+\int_{0}^{t_{1}-t_{m}+d^{\prime}} v_{b}(\tau) d \tau \\
& +\int_{0}^{t_{1}-t_{m}+\left(t_{1}-t_{m}\right)} v_{c}(\tau) d \tau .
\end{aligned}
$$

Now consider the upper limit of the second integral in (45)

$$
\begin{aligned}
t_{1}-t_{m}+d^{\prime} & =t_{1}-t_{m}+t-t_{2} \\
& =t_{1}-t_{m}+t-\left(t_{1}+d_{2}\right)=t-\left(t_{m}+d_{2}\right) \\
& \geq t-t_{1} \quad \text { since } d_{2} \leq\left(t_{1}-t_{m}\right)
\end{aligned}
$$

from (19). For the upper limit of the third integral in (45)

$$
\begin{aligned}
t_{1} & -t_{m}+t_{1}-t_{m} \\
& \geq d_{2}+d_{3} \quad \text { since } d_{2} \leq\left(t_{1}-t_{m}\right) \text { by (19) } \\
& \quad \text { and } d_{3} \leq d_{2} \text { by }(22) \\
& =t_{1}+d_{2}+d_{3}-t_{1} \\
& \geq t-t_{1} \quad \text { since } t \leq t_{1}+d_{2}+d_{3} .
\end{aligned}
$$

We can then rewrite (45) as follows:

$$
\begin{aligned}
c(t)= & c_{a}(t)+c_{b}(t)+c_{c}(t) \\
\geq & \int_{0}^{t-t_{1}} v_{a}(\tau) d \tau+\int_{0}^{t_{1}-t_{m}+d^{\prime}} v_{b}(\tau) d \tau \\
& +\int_{0}^{t_{1}-t_{m}+\left(t_{1}-t_{m}\right)} v_{c}(\tau) d \tau \\
\geq & \int_{0}^{t-t_{1}} v_{a}(\tau) d \tau+\int_{0}^{t-t_{1}} v_{b}(\tau) d \tau \\
& +\int_{0}^{t-t_{1}} v_{c}(\tau) d \tau \operatorname{from}_{(46) \text { and }(47)]}^{t-t_{1}} v(\tau) d \tau=\int_{t_{1}}^{t} v\left(\tau-t_{1}\right) d \tau \\
= & \int_{0}^{t} v(\tau)
\end{aligned}
$$

which shows that playback is also continuous during phase 3 .

Up to now we have proved that playback is continuous from $t=t_{1}$ up to $t=t_{2}+d_{3}$, i.e., covering the period from phase 1 to phase 3 . In the next theorem, we will show that playback is also continuous from the end of phase 3 to the end of the video session.

Theorem 4: Video playback is continuous for the rest of the video session after phase 3, i.e., $c(t) \geq \int_{t_{1}}^{t} v\left(\tau-t_{1}\right) d \tau$, for $t_{2}+d_{3} \leq t \leq L+t_{1}$.

Proof: At any time $t$ after phase 3, the client has received the whole video blocks $B_{1}, B_{2}, C_{1}$, and $C_{2}$. And at the start of phase 3 , i.e., when $t=t_{2}$, the client will start caching data from the static channel for blocks $B_{3}$ and $C_{3}$. Thus, the total amount of continuous data received for slice $\mathrm{B}$, including blocks $B_{1}, B_{2}$ and $B_{3}$, is given by

$$
\begin{aligned}
c_{b}(t)= & \int_{0}^{t_{1}-t_{m}} v_{b}(\tau) d \tau+\int_{t_{1}-t_{m}}^{t_{1}-t_{m}+d_{2}} v_{b}(\tau) d \tau \\
& +\int_{t_{1}-t_{m}+d_{2}}^{t-t_{m}} v_{b}(\tau) d \tau \\
& =\int_{0}^{t-t_{m}} v_{b}(\tau) d \tau .
\end{aligned}
$$


Similarly, the total amount of continuous data received for slice $\mathrm{C}$, including blocks $C_{1}, C_{2}$ and $C_{3}$, is given by

$$
\begin{aligned}
c_{b}(t)= & \int_{0}^{t_{1}-t_{m}} v_{c}(\tau) d \tau+\int_{t_{1}-t_{m}}^{t_{1}-t_{m}+d_{2}} v_{c}(\tau) d \tau \\
& +\int_{t_{1}-t_{m}+d_{2}}^{t-t_{m}} v_{c}(\tau) d \tau \\
= & \int_{0}^{t-t_{m}} v_{c}(\tau) d \tau .
\end{aligned}
$$

Therefore, the total amount of continuous data received by the client is given by

$$
\begin{aligned}
c(t)= & c_{a}(t)+c_{b}(t)+c_{c}(t) \\
= & \int_{0}^{t-t_{1}} v_{a}(\tau) d \tau+\int_{0}^{t-t_{m}} v_{b}(\tau) d \tau+\int_{0}^{t-t_{m}} v_{c}(\tau) d \tau \\
\geq & \int_{0}^{t-t_{1}} v_{a}(\tau) d \tau+\int_{0}^{t-t_{1}} v_{b}(\tau) d \tau \\
& +\int_{0}^{t-t_{1}} v_{c}(\tau) d \tau \quad \because t_{1} \geq t_{m} \\
= & \int_{0}^{t-t_{1}} v(\tau) d \tau=\int_{t_{1}}^{t} v\left(\tau-t_{1}\right) d \tau
\end{aligned}
$$

which shows that playback is continuous after phase 3 until the end of the video session.

Together, Theorems 1-4 establish the fact that playback continuity is guaranteed by the TSP algorithm for the entire duration of the video session.

\section{Performance Evaluation}

In this section, we present simulation results to evaluate and compare the SCP, DCP, the original S\&P [1], and the TSP algorithms investigated in this study. The simulator is developed in $\mathrm{C}++$ using the CNCL simulation library. ${ }^{1}$ The VBR video bit rate traces are measured from 300 DVD videos comprising a wide variety of contents, ranging from full-length movies to documentaries. These video traces exhibit a wide spectrum of properties. For example, the video length ranges from 93-14585 $\mathrm{s}$, and the video bit rate ranges from 1.02-9.85 Mbps. Note that we do not measure the video bit rate directly as most of the DVD bit streams are encrypted. Instead, we measure the I/O activities while playing back the DVD using a hardware MPEG2 decoder. Thus the bit rate profiles not only capture the variations due to video encoding, but also capture the $\mathrm{I} / \mathrm{O}$ behavior of the decoder as well.

The server has a bandwidth of $50 R_{\text {avg }}$ bps and the client an access bandwidth of $2 R_{\text {avg }}$ Mbps, where $R_{\text {avg }}$ is the average bit rate of the VBR-encoded video. Each simulation runs for a

${ }^{1}$ [Online]. Available: http://www.comnets.rwth-aachen.de/doc/cncl.html

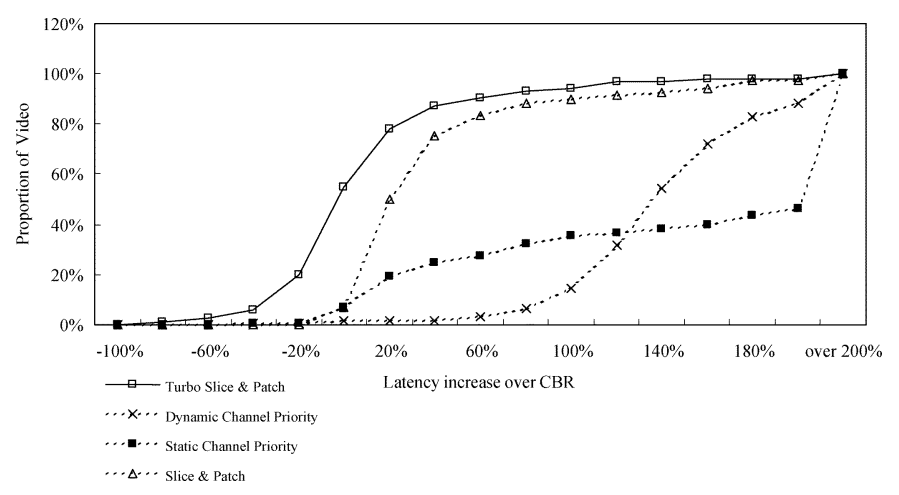

Fig. 5. Comparison of mean latency for all videos.

simulated time of 30 days, with randomized initial conditions. The client arrival rate is 1 request per second. For a video of length $2 \mathrm{~h}$ or $7200 \mathrm{~s}$, this arrival rate represents an average of 7200 concurrent clients in the system.

In configuring the S\&P and the TSP algorithms, which both have a system parameter $R_{\text {cut }}$ that affects the system's performance, we simulate 20 values of $R_{\text {cut }}$ linearly spaced from $R_{\text {avg }}$ to $(4 / 3) R_{\max }$ and select the one that achieves the lowest average latency. Our results in Section VII-C show that while the choice of $R_{\text {cut }}$ is dependent on the video bit rate profile, the sensitivity is relatively modest and thus the simple procedure we employed is sufficient to obtain good results.

\section{A. Average Latency}

Fig. 5 compares the four algorithms' latency for 300 different videos, plotted as a cumulative plot with the horizontal axis representing latency increase over the CBR case. For example, a latency increase of $10 \%$ represents a latency $10 \%$ longer than the latency achieved by the system streaming a CBR video of the same average bit rate and duration. Note that a negative latency increase means that the VBR case achieves latency shorter than the CBR equivalent.

First, among the four algorithms DCP performed worst with increased latency in 295 out of the 300 videos, followed by SCP (279 out of 300) and S\&P (279 out of 300). By contrast, TSP performed significantly better with less than half of the videos (135 out of 300) requiring longer latencies. Second, in terms of variations in latency increases, TSP is also the best with a standard deviation of only $37 \%$. SCP has the worst variation with a standard deviation of $4867 \%$, and a maximum latency increase over $2000 \%$ while the maximum latency of all 300 videos in TSP is only $535 \%$. This shows that the performance of TSP is more robust and less affected by the variations in the video bit rate profile.

SCP's significantly higher variation in latency is due to variations in the bit rate of the videos' initial portion. As the algorithm gives priority to cache from the static channel video data that cannot be used to begin video playback, if the initial portion of the video has a high bit rate, then the dynamic channel will take a longer time to cache sufficient video data to begin playback, thus increasing the latency. By contrast, the DCP, S\&P, and TSP algorithms are less sensitive to this effect because they allocate more bandwidth to cache video data that can be played back immediately. Fig. 6 illustrates this problem by plotting the 


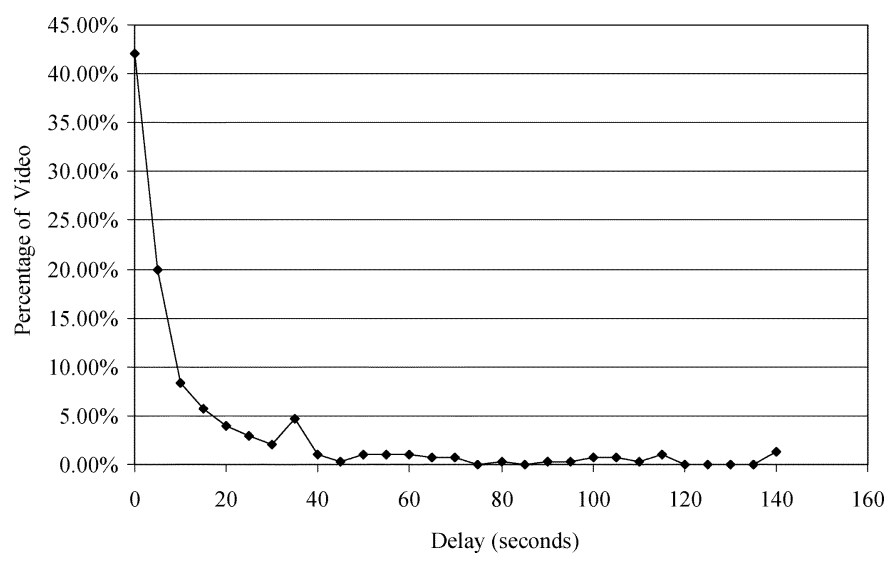

Fig. 6. Playback delay $d_{s}$ for all videos.

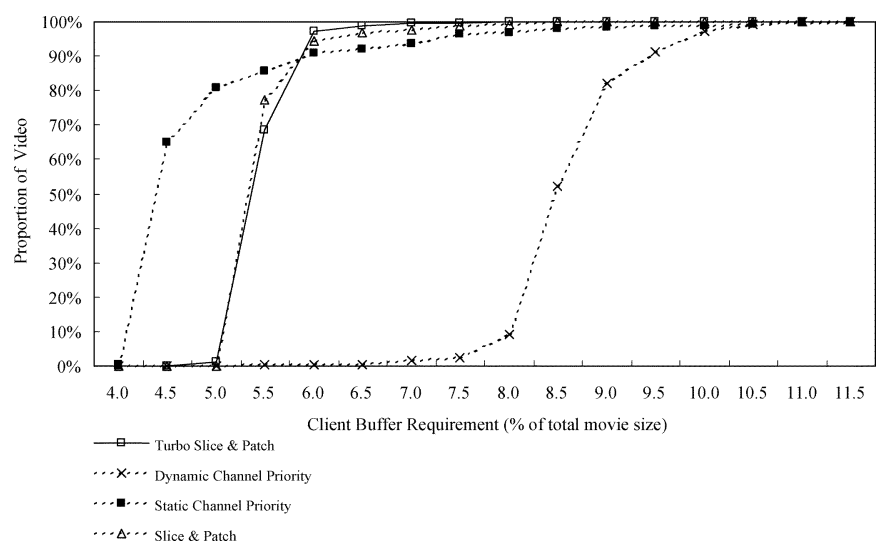

Fig. 7. Comparison of client buffer requirement.

distribution of playback delay of SCP, i.e., the $d_{s}$ in (7). The results show that more than $60 \%$ of videos require a playback delay of $5 \mathrm{~s}$ or more, with a mean as high as $13.9 \mathrm{~s}$. Note that this playback delay adds to the latency experienced by the client regardless of the system load, thus significantly degrading SCP's performance.

Third, comparing DCP with TSP, DCP manages to achieve lower latency than TSP in 6 out of the 300 videos. It is possible to devise a rule based on the ratio of the prefetch latency to the length of $T_{r}$ as an indicator to select between DCP and TSP given a video's bit rate profile. Our experiments show that such threshold-based selection can correctly pick the better performer five out of six times. Nevertheless, such a selection process still relies on the appropriate choice of the threshold and thus the accuracy is not guaranteed. Alternatively, since the selection is an offline process, one can always perform simulations of the two algorithms and pick the one with the best performance for use in the system.

\section{B. Client Buffer Requirement}

Fig. 7 compares the four algorithms' client buffer requirement. These results are generated based on the assumption that there is a server bandwidth of $50 R_{\text {avg }}$ bps. We first observe that the client buffer requirement of TSP is mostly within the range of $5 \%-6.5 \%$ of the video size. The mean client buffer requirement for SCP, DCP, S\&P, and TSP are 5.7\%, 8.6\%, 5.6\%, and

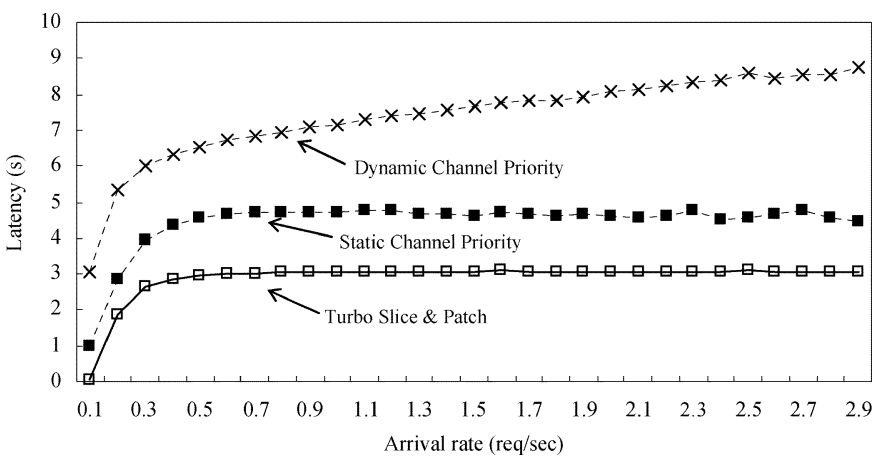

Fig. 8. Comparison of latency for different arrival rates (Video 1).

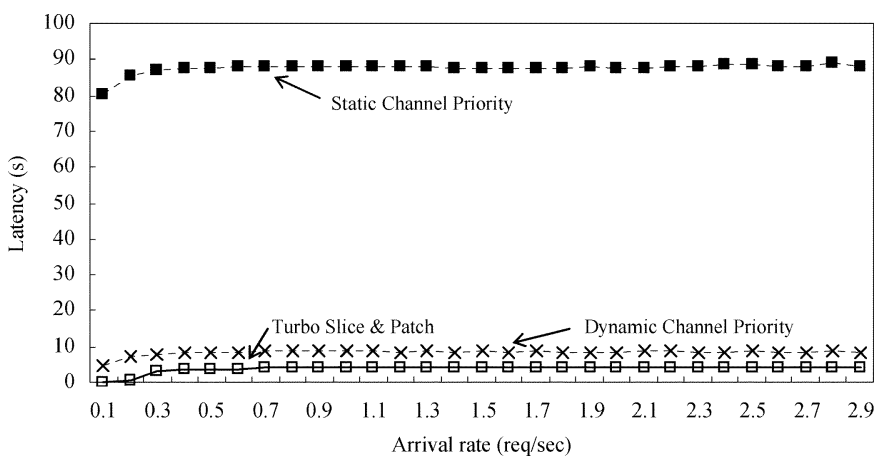

Fig. 9. Comparison of latency for different arrival rates (Video 2).

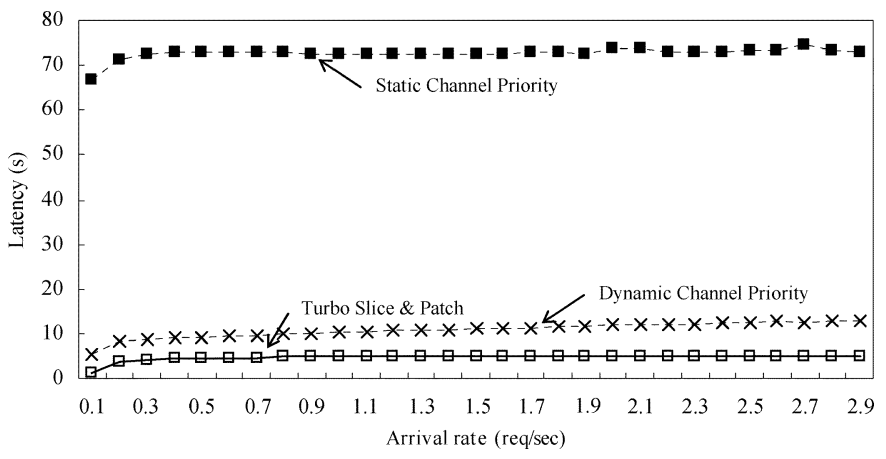

Fig. 10. Comparison of latency for different arrival rates (Video 3).

$5.4 \%$, respectively. For instance, for a video of size $2 \mathrm{~GB}$, the amount of buffer required by TSP will be around $130 \mathrm{MB}$. This can be accommodated by a low-cost harddisk or even stored in memory given the continuous drop in RAM cost. Second, the maximum client buffer requirement over all 300 videos are $11.4 \%, 10.6 \%, 11.4 \%$, and $7.8 \%$ for SCP, DCP, S\&P, and TSP, respectively. Again for a video of size $2 \mathrm{~GB}$, this translates into a buffer requirement of $156 \mathrm{MB}$ for TSP, clearly within the storage limit of even the smallest harddisk.

\section{Performance Impact of the Parameter $R_{\mathrm{cut}}$}

In the previous results we configure $R_{\text {cut }}$ by selecting the best result from 20 samples linearly spaced across the valid range. To further investigate the impact of $R_{\text {cut }}$, we repeat the simulations for 20,40, and 80 samples and find the latency to decrease from $4.59 \mathrm{~s}$ for 20 samples to 4.52 and $4.49 \mathrm{~s}$ for 40 and 80 samples, respectively. Thus evaluating more samples will produce lower latency but the difference quickly diminishes. Nevertheless, as 




Fig. 11. Performance gain on increasing server bandwidth (Video 1).

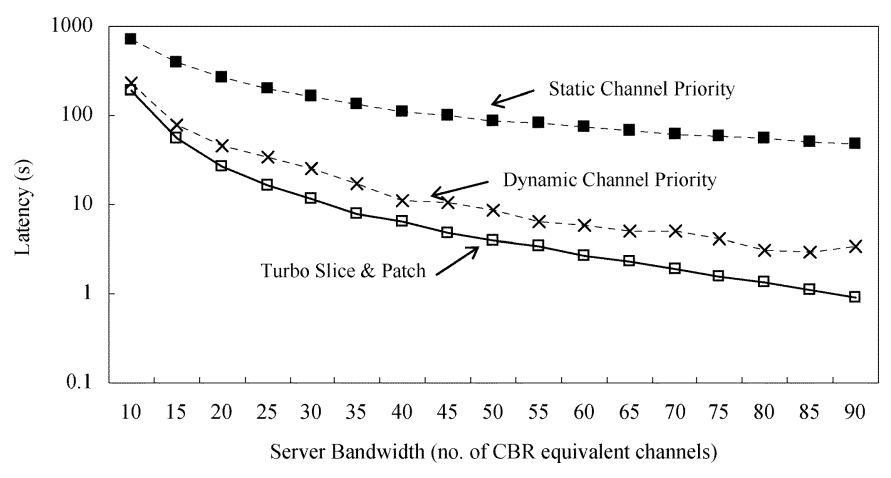

Fig. 12. Performance gain on increasing server bandwidth (Video 2).

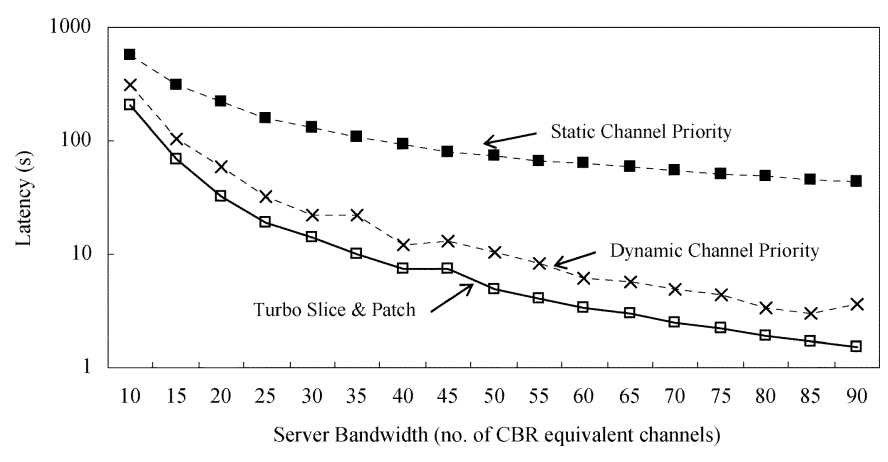

Fig. 13. Performance gain on increasing server bandwidth (Video 3).

the process is performed offline, one could afford to trade off simulation time to obtain better performance.

\section{Latency Versus Arrival Rate}

By fixing the server bandwidth at $50 R_{\text {avg }}$ bps and a maximum of $50 \%$ server bandwidth allocated for static channels, we vary the arrival rate to study the effect on the latency. Figs. 8-10 shows the mean latency versus arrival rate ranging from $1 \times 10^{-2}$ to 3.0 requests per second for three different videos. These three videos are chosen to represent videos from the first quartile (video 1), median (video 2), and third quartile (video 3 ) of the latency distribution. As expected, the latency generally increases with the arrival rate. When the arrival rate increases beyond 0.5 requests per second, the latency of both SCP and TSP level off while the latency of DCP continues to increase. Another observation is that while SCP performs well in video 1, its performance deteriorates significantly when streaming video 2 and video 3. This shows the sensitivity of SCP's performance to the

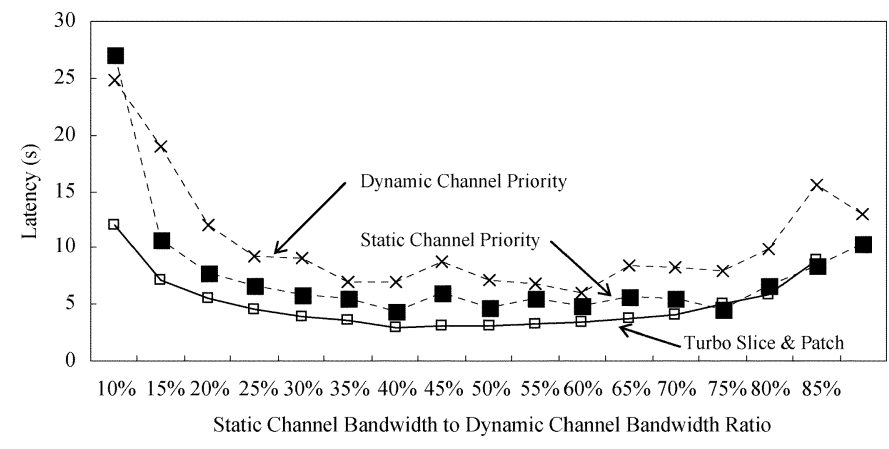

Fig. 14. Effect of server bandwidth partitioning (Video 1).

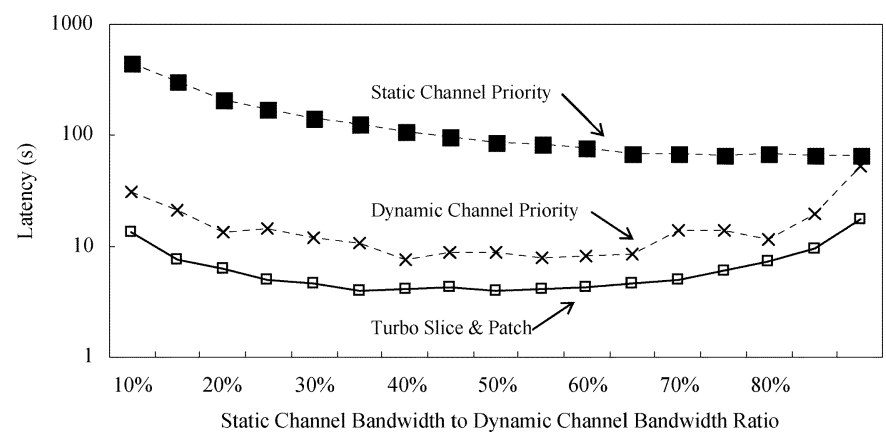

Fig. 15. Effect of server bandwidth partitioning (Video 2).

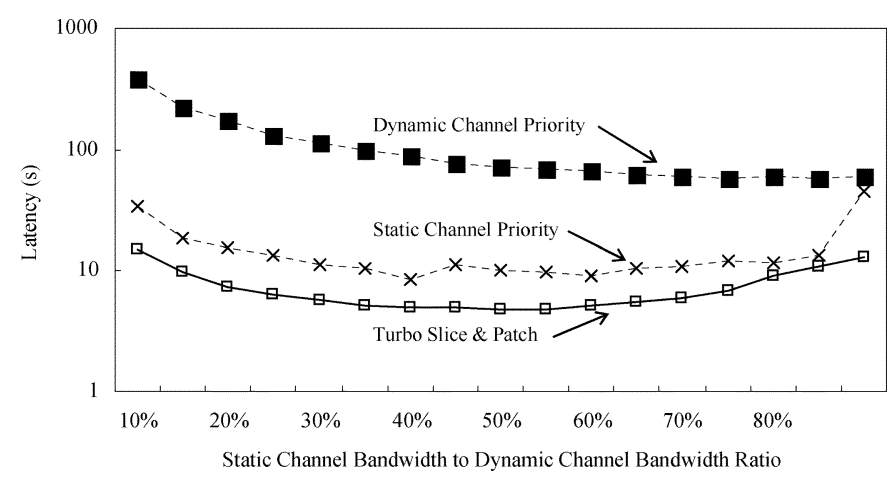

Fig. 16. Effect of server bandwidth partitioning (Video 3).

particular video bit rate profile. The performance of DCP and TSP are more robust in comparison, with TSP achieving the lowest latency in all three cases.

\section{E. Server Bandwidth Comparison}

To investigate the effect of server bandwidth, we fix the arrival rate at 1 request per second and allocate at most 50\% of server bandwidth for static channels. Figs. 11-13 show the mean latency versus server bandwidth for video 1, 2, and 3, respectively. The horizontal axis shows the number of equivalent CBR channels. For example, the value of 50 represents the server bandwidth is $50 R_{\text {avg. }}$. As expected, the mean latency drops when more server bandwidth is available. The results indicate that the latency decreases nearly exponentially when the server bandwidth increases. This suggests that all four algorithms are super-scalar, i.e., the performance increases superlinearly with respect to the resources provided. This is also consistent with the super-scalar property of the original SS-VoD architecture streaming CBR videos [13]. 


\section{F. Bandwidth Partitioning}

To investigate the performance impact of different bandwidth partitioning, we run simulations with a fixed arrival rate of 1 request per second and varying the bandwidth partition ratios. Figs. 14-16 show the latency versus the ratio of static channel bandwidth for Videos 1, 2, and 3, respectively. The horizontal axis is the percentage of bandwidth allocated to static channels. We observe that for TSP, the latency is lowest when half of the bandwidth is assigned to static channels. However, for DCP and $\mathrm{SCP}$, the optimal partition ratio that gives the lowest mean latency is not constant. For example, there are a number of local minima for both algorithms for video 1 . In the other two videos, the latency of SCP decreases with more static channel bandwidth while the optimal partition ratios for DCP are $40 \%$ for both Videos 2 and 3. These results suggest that TSP is significantly simpler to deploy in practice as a bandwidth partition ratio of $50 \%$ can already provide consistent performance. By contrast, the service provider will need to determine and adjust the bandwidth partition ratio for DCP and SCP on a video-byvideo basis to obtain the best performance.

\section{CONCLUSION}

The TSP algorithm investigated in this study addresses two challenges in video streaming. First, TSP employs network multicast to achieve a super-linear scalability that is essential to achieving economy-of-scale in provisioning metropolitan-scale video streaming services. Second, TSP employs a novel threephase S\&P algorithm to support the streaming of VBR-encoded videos with on average only $9 \%$ increase in latency. Given that a previous study has shown that one can achieve the visual quality of CBR-encoded videos using only half the bit rate with VBR encoding, streaming VBR video using TSP in fact requires less resources than streaming CBR videos. With the continuous deployment of multicast in the infrastructure, TSP will serve as a candidate for implementing the future metropolitan video streaming services.

\section{ACKNOWLEDGMENT}

The authors would like to thank the anonymous reviewers for their thoughtful comments and suggestions in improving this paper.

\section{REFERENCES}

[1] C. W. Kong and J. Y. B. Lee, "Slice-and-patch—an algorithm to support VBR video streaming in a multicast-based video-on-demand system," in Proc. 2002 Int. Conf. Parallel Distrib. Syst., Dec. 2002, pp. 391-397.

[2] A. Dan, D. Sitaram, and P. Shahabuddin, "Scheduling policies for an on-demand video server with batching," in Proc. 2nd ACM Multimedia, 1994, pp. 15-23.

[3] H. Shachnai and P. S. Yu, "Exploring waiting tolerance in effective batching for video-on-demand scheduling," in Proc. 8th Israeli Conf. Comput. Syst. Softw. Eng., Jun. 1997, pp. 67-76.

[4] V. O. K. Li, W. Liao, X. Qui, and E. W. M. Wong, "Performance model of interactive video-on-demand systems," Proc. IEEE JSAC, vol. 14, no. 6, pp. 1099-1109, Aug. 1996

[5] W. Liao and V. O. K. Li, "The split and merge protocol for interactive video-on-demand," IEEE Multimedia, vol. 4, no. 4, pp. 51-62, Apr. 1997.
[6] K. A. Hua, Y. Cai, and S. Sheu, "Patching: a multicast technique for true video-on-demand services," in Proc. 6th Int. Conf. Multimedia, Sep. 1998, pp. 191-200.

[7] Y. Cai, K. Hua, and K. Vu, "Optimizing patching performance," in Proc. SPIE/ACM Conf. Multimedia Comput. Netw., Jan. 1999, pp. 204-215.

[8] S. W. Carter, D. D. E. Long, K. Makki, L. M. Ni, M. Singhal, and N. Pissinou, "Improving video-on-demand server efficiency through stream tapping," in Proc. 6th Int. Conf. Comput. Commun. Netw., Sep. 1997, pp. 200-207.

[9] D. Saparilla, K. W. Ross, and M. Reisslein, "Periodic broadcasting with VBR-encoded video," in Proc. IEEE Infocom, New York, Mar. 1999, pp. 464-471.

[10] S. Sen, G. Lixin, and D. Towsley, "Frame-based periodic broadcast and fundamental resource tradeoffs," in Proc. IEEE Int. Conf. Perform., Comput., Commun., 2001, pp. 77-83.

[11] T. C. Chiueh and C. H. Lu, "A periodic broadcasting approach to video-on-demand service," in Proc. SPIE, vol. 2615, 1996, pp. $162-169$.

[12] A. Hu, I. Nikolaidis, and P. van Beek, "On the design of efficient video-on-demand broadcast schedules," in Proc. 7th Int. Symp. Model., Anal. Simulation Comput. Telecomm. Syst., 1999, pp. 262-269.

[13] J. Y. B. Lee and C. H. Lee, "Design, performance analysis, and implementation of a super-scalar video-on-demand system," IEEE Trans. Circuits Syst. Video Technol., vol. 12, no. 11, pp. 983-997, Nov. 2002.

[14] W. S. Tan, N. Duong, and J. Princen, "A comparison study of variable-bit rate versus fixed-bit rate video transmission," in Proc. Aus. Broadband Switch. Services Symp., 1991, pp. 134-141.

[15] W. Feng and S. Sechrest, "Smoothing and buffering for the delivery of pre-recorded video," in Proc. ISET/SPIE Multimedia Comput. Netw., Feb. 1995, pp. 234-244.

[16] J. D. Salehi, Z. L. Zhang, J. F. Kurose, and D. Towsley, "Supporting stored video: reducing rate variability and end-to-end resource requirements through optimal smoothing," in Proc. ACM SIGMETERICS, May 1996, pp. 222-231.

[17] W. Feng, F. Jahanian, and S. Sechrest, “ An optimal bandwidth allocation strategy for the delivery of compressed prerecorded video," in $A C M$ Multimedia Syst. J., vol. 5, Sep. 1995, pp. 297-309.

[18] H. L. Lai, J. Y. B. Chen, and L. K. Chen, " A monotonic-decreasing rate scheduler for variable-bit-rate video streaming," IEEE Trans. Circuits Syst. Video Technol., vol. 15, no. 2, pp. 221-231, Feb. 2005.

[19] W. Feng, W. Mishra, and W. Ramakishnan, "A comparison of bandwidth smoothing techniques for the transmission of pre-recorded compressed video," in Proc. INFOCOM, vol. 1, 1997, pp. 58-66.

[20] D. Y. Lee and H. Y. Yeom, "Tip prefetching: dealing with the bit rate variability of video streams," in Proc. IEEE ICMCS, vol. II, 1999, pp. $352-356$.

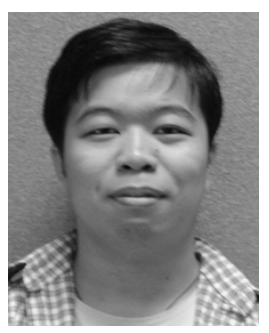

Chun-Wai Kong received the B.Eng and M.Phil degrees in information engineering from the Chinese University of Hong Kong, in 2000 and 2002, respectively.

He was a member of the Multimedia Communications Laboratory and participated in the research of algorithms and systems for multicast video streaming.

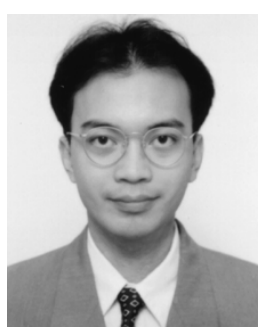

Jack Y. B. Lee (M'95-SM'03) received the B.Eng and $\mathrm{Ph} . \mathrm{D}$ degrees in information engineering from the Chinese University of Hong Kong, Hong Kong, SAR, in 1993 and 1997, respectively.

He participated in the research and development of video streaming systems at the Chinese University of Hong Kong from 1997 to 1998 where he and his team developed novel parallel video server architectures for building cost-effective, scalable and fault-tolerant video-on-demand systems. This work had resulted in numerous publications, two U.S. patents, and the technologies are subsequently transferred to a spin-off technology company for commercialization. He was a faculty member at the Department of Computer Science at the Hong Kong University of Science and Technology from 1998-1999, and in 1999 he joined the Department of Information Engineering at the Chinese University of Hong Kong to establish the Multimedia Communications Laboratory to spearhead research in distributed multimedia systems, fault-tolerant systems, peer-to-peer systems, multicast communications, and Internet computing. 


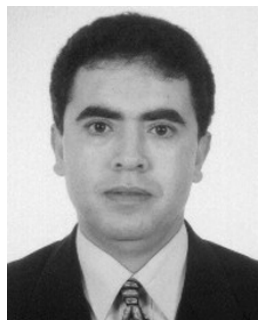

Mounir Hamdi (S'89-M'90) received the B.S. degree in computer engineering (with distinction) from the University of Louisiana, Lafayette, in 1985 and the M.S. and the Ph.D. degrees in electrical engineering from the University of Pittsburgh, Pittsburgh, PA, in 1987 and 1991, respectively.

He has been a Faculty Member in the Department of Computer Science, Hong Kong University of Science and Technology since 1991, where he is now a Professor of Computer Science and the Director of the Computer Engineering Program that has around 350 undergraduate students. From 1999 to 2000, he held Visiting Professor positions at Stanford University, Stanford, CA, and the Swiss Federal Institute of Technology, Lausanne, Switzerland. His general areas of research are in high-speed packet switches/routers and all-optical networks, in which he has published more than 200 research publications, received numerous research grants, supervised some 20 postgraduate students, and for which he has served as consultant to various international companies. Currently, he is working on high-speed networks including the design, analysis, scheduling, and management of high-speed switches/routers, wavelength-division multiplexing (WDM) networks/switches, and wireless networks. He is currently leading a team that is designing one the highest capacity chip sets for terabit switches/routers. This chip set is targeted toward a $256 \times 256$ OC-192 switch, and includes a crossbar fabric chip, a scheduler/arbiter chip, and traffic management chip. In addition to his commitment to research and professional service, he is also a dedicated teacher.

Dr. Hamdi has been on the Editorial Board of IEEE TRANSACTIONS ON COMmunications, IEEE COMMUNiCATION MAGaZine, Computer Networks, WiRELESS COMMUNICATIONS and Mobile Computing, and Parallel Computing, and has been on the program committees of more than 70 international conferences and workshops. He was a Guest Editor of IEEE COMMUNICATIONS MAGAZINE, guest editor-in-chief of two special issues of IEEE JOURNAL on SElECted AREAS of COMMUNiCATIONS, and a Guest Editor of Optical Networks Magazine, and has chaired more than five international conferences and workshops including the IEEE GLOBECOM/ICC Optical networking workshop, the IEEE ICC High-speed Access Workshop, and the IEEE IPPS HiNets Workshop. He is/was the Chair of IEEE Communications Society Technical Committee on Transmissions, Access and Optical Systems, and Vice-Chair of the Optical Networking Technical Committee, as well as member of the ComSoc technical activities council. He is/was on the technical program committees of more than 100 international conferences and workshops. He received the Best Paper Award at the International Conference on Information and Networking in 1998 out of 152 papers. He also supervised the best Ph.D. paper award among all universities in Hong Kong. He received the Best Ten Lecturer's Award (through university-wide student voting for all university faculty held once a year), the Distinguished Engineering Teaching Appreciation Award from the Hong Kong University of Science and Technology, and various grants targeted toward the improvement of teaching methodologies, delivery and technology. He is a member of ACM.

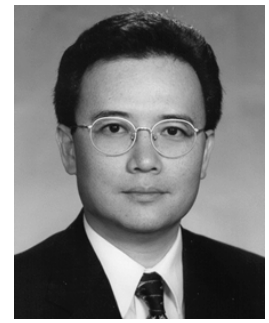

Victor O. K. Li (S'80-M'81-SM'86-F'92) was born in Hong Kong in 1954. He received the S.B., S.M., E.E., and Sc.D. degrees in electrical engineering and computer science from the Massachusetts Institute of Technology, Cambridge, in 1977, 1979, 1980, and 1981, respectively.

He joined the University of Southern California (USC), Los Angeles, in February 1981, and became Professor of Electrical Engineering and Director of the USC Communication Sciences Institute. Since September 1997, he has been with the University of Hong Kong, Hong Kong, where he is Chair Professor of Information Engineering at the Department of Electrical and Electronic Engineering, and Managing Director of Versitech Ltd., the technology transfer and commercial arm of the University. He also serves on various corporate boards. His research is in information technology, including high-speed communication networks, wireless networks, and Internet technologies and applications. He is a Principal Investigator of the Area of Excellence in Information Technology funded by the Hong Kong government. Sought by government, industry, and academic organizations, he has lectured and consulted extensively around the world He chaired the Computer Communications Technical Committee of the IEEE Communications Society (1987-1989), and the Los Angeles Chapter of the IEEE Information Theory Group (1983-1985). He co-founded the International Conference on Computer Communications and Networks (IC3N), and chaired its Steering Committee 1992-1997. He also chaired various international workshops and conferences, including, most recently, IEEE INFOCOM 2004. He was appointed to the Hong Kong Information Infrastructure Advisory Committee by the Chief Executive of the Hong Kong Special Administrative Region. He also serves on the Innovation and Technology Fund (Electronics) Vetting Committee, the Small Entrepreneur Research Assistance Programme Committee, the Engineering Panel of the Research Grants Council, and the Task Force for the Hong Kong Academic and Research Network (HARNET) Development Fund of the University Grants Committee. He was a Distinguished Lecturer at the University of California at San Diego, at the National Science Council of Taiwan, and at the California Polytechnic Institute. He has also delivered keynote speeches at many international conferences.

Prof. Li has received numerous awards, including, most recently, the Outstanding Researcher Award of the University of Hong Kong, the KC Wong Education Foundation Lectureship, the Croucher Foundation Senior Research Fellowship, and the Bronze Bauhinia Star, Government of the Hong Kong Special Administrative Region, China. He has served as an Editor of IEEE Network, IEEE JOURNAL ON SELECTED AREAS IN COMMUNICATIONS (JSAC) Wireless Communications Series, and Telecommunication Systems. He also guest edited special issues of IEEE JSAC, Computer Networks and ISDN Systems, and KICS/IEEE Journal of Communications and Networking. He is now serving as an editor of ACM/Kluwer Wireless Networks and IEEE Communications Surveys and Tutorials. 